\title{
Dissipative multi-resonator acoustic metamaterials for impact force mitigation and collision energy absorption
}

\author{
Q.Q Li ${ }^{\mathrm{a}}$, Z.C. $\mathrm{He}^{\mathrm{a}^{*}}$, Eric $\mathrm{Li}^{\mathrm{b} *}$ \\ ${ }^{a}$ State Key Laboratory of Advanced Design and Manufacturing for Vehicle Body, Hunan University, \\ Changsha, 410082 P. R. China \\ ${ }^{b}$ School of Science, Engineering \& Design, Teesside University, Middlesbrough, UK
}

\begin{abstract}
In this paper, we study the interesting phenomena of stress waves attenuation and impact energy dissipation through the application of multi-resonator dissipative acoustic metamaterials (DAMs) with Kelvin-Voigt-type (KVT), Maxwell-type (MT) and Zener-type (ZT) oscillators. The theory analyses show these microstructures have broad negative effective mass frequency regions and high effective metadamping coefficient. The numerical results prove that the damping characteristic can gather the multi band gaps of the elastic multi-resonator metamaterial together. KVT oscillators present the best performances in impact load mitigation and collision energy dissipation among these DAMs. With very small damping coefficients, the attenuation and dissipation effects of MT models are poor, and the performances of ZT and KVT models are similar. Through properly tailoring the damping, the performances of KVT and MT are significantly improved. Meanwhile, the influence of damping on the performances of ZT oscillators is less compared with KVT and MT models.
\end{abstract}

Key words: dissipative acoustic metamaterials; multi-resonators; metadamping;

\footnotetext{
* Corresponding author.

E-mail address: hezhicheng815@hnu.edu.cn (Z.C. He); q.li@tees.ac.uk (Eric Li)
}

\section{NOMENCLATURE}

$m_{1}, m_{2}, m_{3}, m_{4}$ and $m_{5}$

$u_{1}, u_{2}, u_{3}, u_{4}$ and $u_{5}$

$k_{2}, k_{3}, k_{4}$ and $k_{5}$
Outer and inner masses of microstructure

Displacement of each rigid mass

Stiffness of each internal spring 
$u_{22}, u_{32}, u_{42}$ and $u_{52}$

$c_{2}, c_{3}, c_{4}$ and $c_{5}$

$\tau_{i}=c_{i} / \sqrt{k_{i} m_{i}}(i=2, \ldots, 5)$

$\omega / \omega_{2}$ and $\omega / \omega_{4}$

$m_{\text {eff }} / m_{s t}$

$c_{\text {eff }}$
Additional degree of movements in MT and ZT microstructures

Damping coefficients

Dimensionless damping coefficient

Dimensionless frequencies of rigid masses $m_{2}$ and $m_{4}$

Dimensionless effective mass

Effective metadamping coefficient

\section{Introduction}

Metamaterials are the artificial composite materials which have exceptional properties that are not found in nature [1-7]. The superior properties of metamaterials are determined by special arrangement of microstructure instead of chemical composition. Therefore, metamaterials provide us the new avenues for the design of novel materials and structures. Among these metamaterials, photonic crystals $[8,9]$, magnetic metamaterials [10-12] as well as acoustic metamaterials (AMs) [13-21] are widely studied.

In this work, we focus our studies on the attenuation effects of AMs which received substantial attentions in the past, and previous researches on AMs include multiple fields, such as acoustics attenuation [22, 23], noise control [24-29], invisibility cloak [30] and energy absorption [31, 32]. A kind of one-dimensional resonant AM [33] was presented, and its left-handed propagation property of acoustic waves was also demonstrated. Two kinds of plate-type AMs [34] were studied through the theoretical and numerical analyses, and these metamaterial plates achieve high voice propagation attenuation. One new typical of AM was studied [35], and through the translational and rotational motions of the oscillators, the properties of negative mass and negative modulus are obtained. The attenuation effects of impact load are achieved with elastic metamaterials, meta-lattice sandwich structure and Maxwell-type oscillators [36-38], respectively. The transmission loss of a membrane-type locally resonant AM with added ring masses was studied [39]. Through finite element simulations and experimental tests, it is found that this 
metamaterial exhibits high attenuation effect of mechanical waves over a wide frequency spectrum region. The width of the band gaps depends on the added rings and the distribution of masses between the ring masses and the inner mass seriously. A new multi-frequency resonator metamaterial beam to realize the broadband vibration absorption was presented [40]. Based on the theoretical and numerical analyses, two stopbands are exhibited by this periodic structure. With solid viscoelastic elements, a metamaterial which has the controllable properties was developed [41]. By connecting the mass to the viscoelastic support structure, this system shows the property of negative effective mass. Moreover, with a suitable local control scheme, this system can provide both the negative effective stiffness and the negative effective mass.

The microstructures of AMs exhibit some form of resonance characteristics [42], and these characteristics determine their macro properties. Therefore, the design of microstructures of AMs is crucial to their performance. It is well known that the broadband attenuation can be realized by the multi-resonator microstructures. For example, the double-resonator AMs [43-45] exhibit broader band gap regions than those of the single-resonator AMs, and the mechanical waves cannot propagate in their attenuation frequency ranges. The attenuation mechanism of AMs is to store and reflect the mechanical wave energy by means of local resonance of oscillators. It should be noted that the wave energy is not absorbed. The reason is that the damping property is not considered in the AMs. In fact, it is difficult to find pure elastic resonators because of the widespread damping. Damping is the intrinsic property of the materials, and its characteristic directly affects the absorption of energy. The dissipation capacity of AMs can be greatly improved through the application of the damping on the resonators [46, 47], which achieves better energy absorption effect than the traditional damping structure. However, damping compresses the motions of the resonators, and the attenuation effect of DAMs is generated based on the local resonance of oscillators. The application of damping may reduce the attenuation effects of the oscillators while increasing their dissipation effects. Therefore, it is necessary to conduct a detailed study to investigate the effect of damping on the 
performance of AMs.

In an early study of DAMs, the interactions of resonant motions and viscoelastic effects of DAMs $[48,49]$ was utilized to realize broadband attenuation. Numerical results of the transient blast wave model prove that the broadband waves can be almost completely mitigated with this DAM. The theoretical studies of the propagation characteristics of mechanical waves in the elastic and viscoelastic two-dimensional phononic crystal [50] were carried out, and wide band gaps are achieved by these metamaterials. Based on the Bloch's theorem, the dispersion relation of a local resonance metamaterial with viscoelastic damping was derived [51]. The high dissipation property throughout the frequency spectrum is achieved by the resonators and dampers, which is called the metadamping. The distributed inertial damper [52] was presented to achieve wide bandwidth energy absorption, and a beam structure with the tensile load was applied to prove the efficiency of this distributed damper. The damped spring mass absorber system [53] was applied on the beam structure to attenuate the mechanical waves. With multiple resonant frequencies and high damping loss factor, this system can attenuate the broadband waves efficiently. The combination of a high rigid backbone structure and a viscoelastic resonant lattice was showed [54]. In this system, this chiral topology metamaterial pumps the vibration energy away based on the resonant lattice and dissipates it through the viscoelastic damping. In addition, finite element analyses and experimental tests were applied to demonstrate its high attenuation effect.

In this paper, we investigate the attenuation and dissipation characteristics of DAMs with KVT, MT and ZT multi-resonator microstructures, respectively. Regarding Section 2, the working mechanisms of these three DAMs are revealed based on the motion ratios of inner masses to outer mass. The DAMs are homogenized as the effective mediums, from which the attenuation and dissipation effects of mechanical waves can be quantitatively explained by the negative effective mass and the effective metadamping. The high damping coefficients alleviate the intensity of resonance motions of KVT and ZT oscillators, and they improve the dissipation effects of these microstructures. However, the resonance movements and 
dissipative characteristics of MT model improve first and then decrease with the increase of damping coefficients. In Sections 3 and 4, the impact wave model and the sandwich panel crash model are analyzed to study the performance of these three DAMs. The attenuation of dynamic load, structural deformation and structural stress as well as the dissipation of impact energy of these DAMs are achieved. In addition, the multi band gaps of the elastic multi-resonator microstructure can be combined into one wide band gap through the application of damping. KVT presents the best attenuation and dissipation effects among these damped models. With the very small damping, ZT and KVT have similar impact results, and the performances of MT model are poor. In addition, a reasonable damping can significantly improve the attenuation and dissipation effects of KVT and MT, and the influence of damping on the performances of ZT is less. Finally, the conclusions are summarized in Section 5.

\section{Microstructure of dissipative AM}

In the non-dissipative systems, the energy carried by mechanical waves is actually temporarily stored in the resonators, and it is not absorbed. However, the damped metamaterials can significantly absorb the energy based on their dissipation effects. In this section, four damping elements are introduced into the multi-resonators microstructure. The combination of damping characteristic and negative mass property turns the multiple band gaps into a complete wide frequency mitigation region.

\subsection{KVT multi-resonators microstructure}

In this section, the mitigation and dissipation characteristics of the damped multi-resonator microstructures are briefly presented. Fig. 1(a) shows the dissipative KVT multi-resonators microstructure. This microstructure is composed of five masses, four dashpots and four springs. The resonator masses are $m_{1}, m_{2}, m_{3}, m_{4}$ and $m_{5}$, and their displacements are $u_{1}, u_{2}, u_{3}, u_{4}$ and $u_{5} . c_{2}$ and $k_{2}$ denote the damping coefficient and the spring stiffness which connect the left middle oscillator and the left inner resonator. $c_{3}$ and $k_{3}$ are the damping coefficient and the spring stiffness between the 
left middle mass and the outer mass. $c_{4}, c_{5}, k_{4}$ and $k_{5}$ represent the damping coefficients and the spring stiffness between the right middle mass, the right inner mass and the outer mass. The dissipative one dimensional lattice system, which is composed of the KVT multi-resonators microstructures, is shown in Fig. 1(b). Based on the Bloch-Floquet theory, the harmonic motion of the $(j+n)$ th unit cell in the one dimensional lattice system can be expressed as Eq. (1).

$$
u_{\lambda}^{(j+\mathrm{n})}=\widehat{u}_{\lambda} e^{i(q x+n q a-\omega t)}
$$

where $u_{\lambda}^{(j+\mathrm{n})}$ is the displacement of oscillator $\lambda$ in the $(j+n)$ th unit cell, $\hat{u}_{\lambda}$ is the amplitude of the displacement of oscillator $\lambda, \omega$ is the angular frequency, $a$ is the lattice constant and $q$ is the wavenumber.

Due to the periodicity of the one dimensional lattice system, we study the properties of the entire metamaterials by learning the characteristics of individual microstructure. As shown in Fig. 1(a) and (b), the external force $F^{(j)}$ acting on the $j$ th KVT microstructure can be seen as $k_{1}\left(u_{1}^{(j-1)}+u_{1}^{(j+1)}-2 u_{1}^{(j)}\right)$. Therefore, the external force is also the harmonic load, and it can be expressed as follows.

$$
F^{(j)}=F_{0} e^{i(q x-\omega t)}
$$

where $F_{0}$ is the amplitude of the harmonic load. Then, the governing equations of a single KVT microstructure can be written as Eq. (3) based on Newton's second law.

$$
\begin{aligned}
& m_{2} \ddot{u}_{2}=k_{2}\left(u_{3}-u_{2}\right)+c_{2}\left(\dot{u}_{3}-\dot{u}_{2}\right) \\
& m_{3} \ddot{u}_{3}=k_{2}\left(u_{2}-u_{3}\right)+k_{3}\left(u_{1}-u_{3}\right)+c_{2}\left(\dot{u}_{2}-\dot{u}_{3}\right)+c_{3}\left(\dot{u}_{1}-\dot{u}_{3}\right) \\
& m_{1} \ddot{u}_{1}=F+k_{3}\left(u_{3}-u_{1}\right)+k_{5}\left(u_{5}-u_{1}\right)+c_{3}\left(\dot{u}_{3}-\dot{u}_{1}\right)+c_{5}\left(\dot{u}_{5}-\dot{u}_{1}\right) \\
& m_{4} \ddot{u}_{4}=k_{4}\left(u_{5}-u_{4}\right)+c_{4}\left(\dot{u}_{5}-\dot{u}_{4}\right) \\
& m_{5} \ddot{u}_{5}=k_{5}\left(u_{1}-u_{5}\right)+k_{4}\left(u_{4}-u_{5}\right)+c_{5}\left(\dot{u}_{1}-\dot{u}_{5}\right)+c_{4}\left(\dot{u}_{4}-\dot{u}_{5}\right)
\end{aligned}
$$

Then, the following equation can be captured. 


$$
\left[\begin{array}{lll}
A_{11} & A_{12} & A_{13} \\
A_{21} & A_{22} & A_{23} \\
A_{31} & A_{32} & A_{33} \\
A_{41} & A_{42} & A_{43} \\
A_{51} & A_{52} & A_{53}
\end{array}\right]\left\{\begin{array}{c}
\omega^{2} \\
\omega \\
1
\end{array}\right\}=\left\{\begin{array}{l}
0 \\
0 \\
0 \\
0 \\
0
\end{array}\right\}
$$

in which

$$
\begin{aligned}
& A_{11}=m_{2} \widehat{u}_{2}, A_{21}=m_{3} \widehat{u}_{3}, A_{31}=m_{1} \widehat{u}_{1}, A_{41}=m_{4} \widehat{u}_{4}, A_{51}=m_{5} \widehat{u}_{5}, \\
& A_{12}=-i \tau_{2} \sqrt{k_{2} m_{2}}\left(\widehat{u}_{3}-\widehat{u}_{2}\right), A_{22}=-i\left[\tau_{3} \sqrt{k_{3} m_{3}}\left(\hat{u}_{1}-\widehat{u}_{3}\right)+\tau_{2} \sqrt{k_{2} m_{2}}\left(\hat{u}_{2}-\hat{u}_{3}\right)\right] \text {, } \\
& A_{32}=-i\left[\tau_{3} \sqrt{k_{3} m_{3}}\left(\hat{u}_{3}-\widehat{u}_{1}\right)+\tau_{5} \sqrt{k_{5} m_{5}}\left(\hat{u}_{5}-\widehat{u}_{1}\right)\right], \quad A_{42}=-i \tau_{4} \sqrt{k_{4} m_{4}}\left(\hat{u}_{5}-\widehat{u}_{4}\right), \\
& A_{52}=-i\left[\tau_{4} \sqrt{k_{4} m_{4}}\left(\hat{u}_{4}-\hat{u}_{5}\right)+\tau_{5} \sqrt{k_{5} m_{5}}\left(\hat{u}_{1}-\hat{u}_{5}\right)\right], A_{13}=k_{2}\left(\hat{u}_{3}-\widehat{u}_{2}\right), \\
& A_{23}=k_{3}\left(\hat{u}_{1}-\widehat{u}_{3}\right)+k_{2}\left(\hat{u}_{2}-\widehat{u}_{3}\right), A_{33}=k_{3}\left(\hat{u}_{3}-\widehat{u}_{1}\right)+k_{5}\left(\hat{u}_{5}-\widehat{u}_{1}\right)+F_{o}, \\
& A_{43}=k_{4}\left(\hat{u}_{5}-\widehat{u}_{4}\right), A_{33}=k_{4}\left(\hat{u}_{4}-\hat{u}_{5}\right)+k_{5}\left(\hat{u}_{1}-\widehat{u}_{5}\right)
\end{aligned}
$$

and $\tau_{i}=c_{i} / \sqrt{k_{i} m_{i}} \quad(i=2, \ldots, 5)$ is defined as the dimensionless damping coefficient.

To reveal the working mechanism of KVT multi-resonators microstructure, this microstructure is equivalent to the effective mass and the effective metadamping. Based on Newton's second law, equation $F=m_{e f f} \ddot{u}_{1}+c_{e f f} \dot{u}_{1}$ must be satisfied, where $m_{\text {eff }}$ is the effective mass, $c_{\text {eff }}$ is the effective metadamping coefficient. A large $c_{\text {eff }}$ implies a high dissipation effect of DAMs. Then, the following Eq. (5) can be obtained.

$$
F_{0}=-\widehat{u}_{1}\left(m_{e f f} \omega^{2}+i c_{e f f} \omega\right)
$$



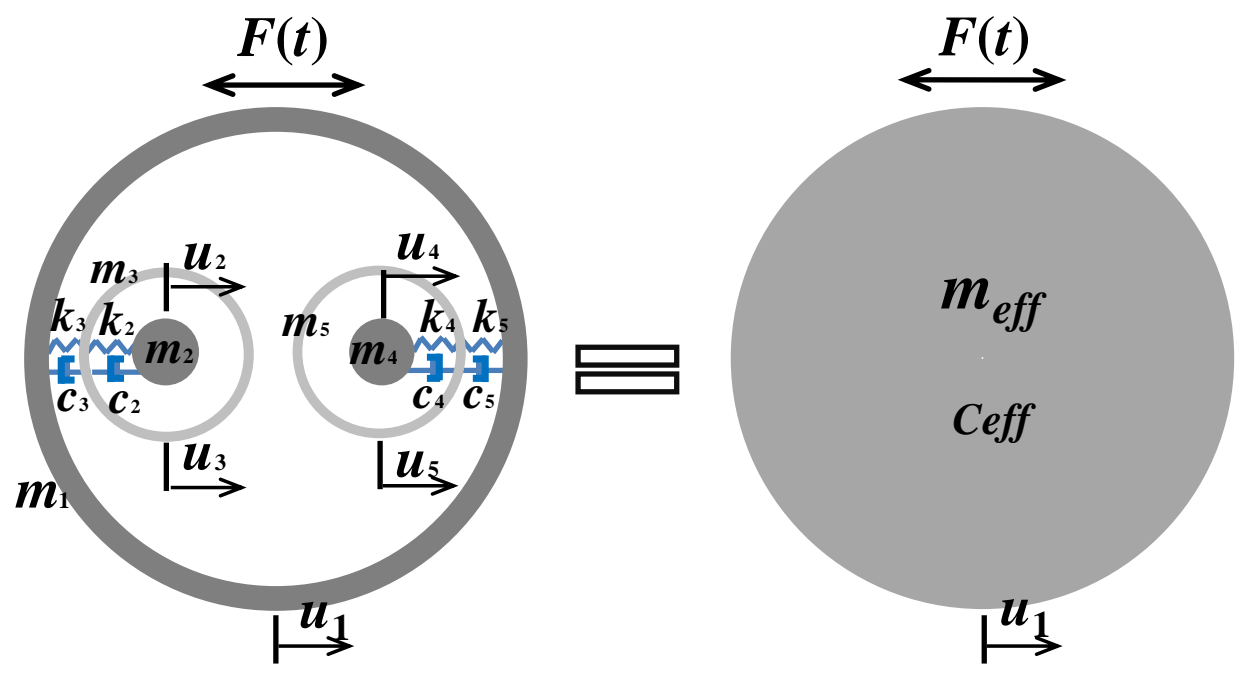

(a)
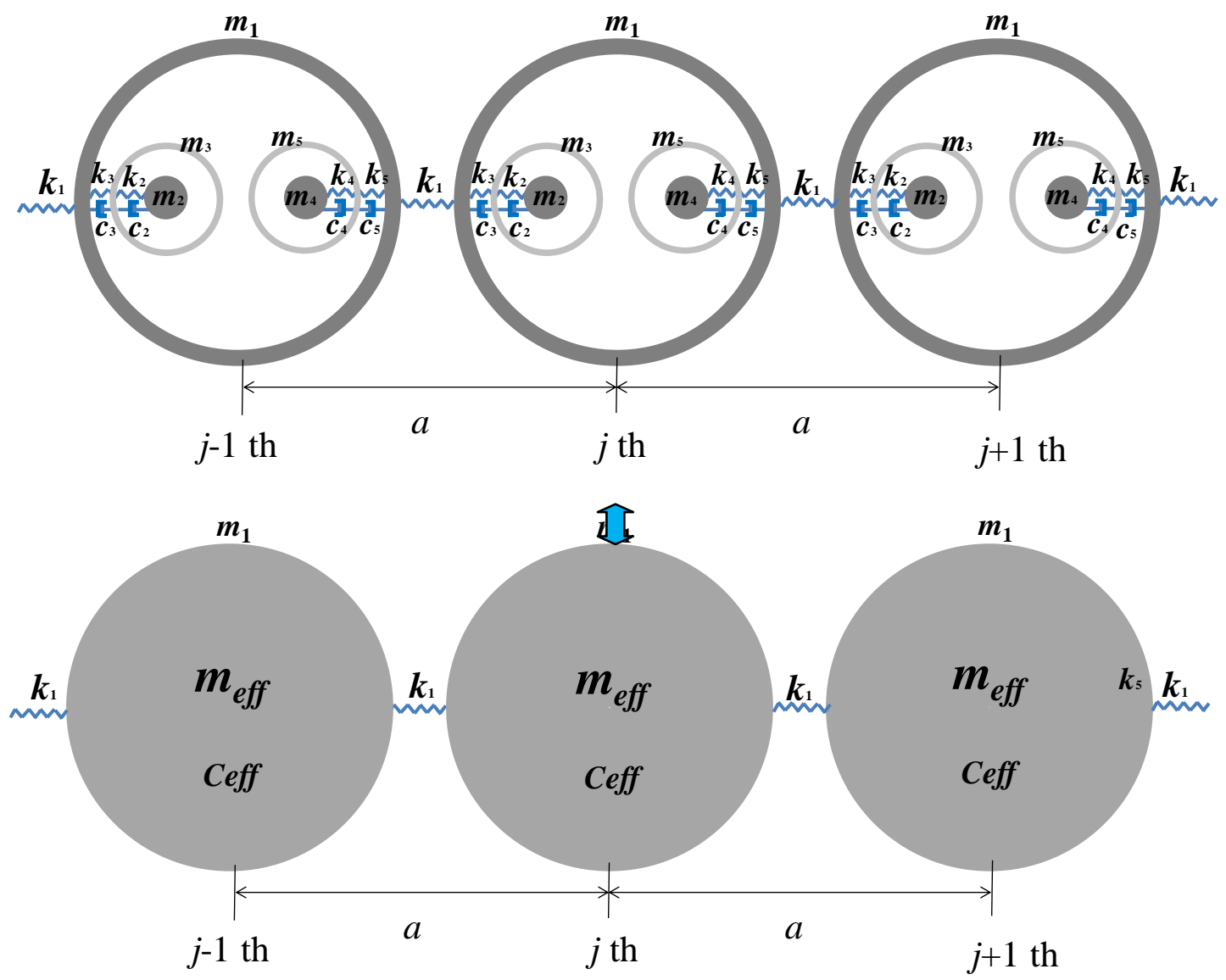

(b)

Fig. 1. (a) KVT multi-resonators microstructure, (b) Dissipative one dimensional KVT multi-resonator lattice system.

Surface plots of dimensionless effective mass $m_{\text {eff }} / m_{s t}$ and effective metadamping coefficient $c_{\text {eff }}$ against dimensionless frequencies $\omega / \omega_{2}$ and $\omega / \omega_{4}$ are shown in Fig. 2. In Fig. 2, the spring stiffness and rigid mass ratios are set as $k_{2} / k_{3}=0.25, k_{4} / k_{5}=0.25$, 
$m_{2} / m_{3}=2.5, m_{3} / m_{1}=2, m_{4} / m_{5}=2.5$ and $m_{5} / m_{1}=2$, respectively. The dimensionless damping coefficients of $\tau_{4}$ and $\tau_{5}$ are set as 0.01 . Sensitivity analyses are carried out to study how these damping parameters affect the band gaps of $m_{\text {eff }} / m_{s t}$ and $c_{\text {eff. }}$. Because of the symmetry of the microstructure, the influences of $\tau_{2}$ and $\tau_{3}$ on the performances of KVT are similar to those of $\tau_{4}$ and $\tau_{5}$. Therefore, only parameters $\tau_{2}$ and $\tau_{3}$ are analyzed. Figs. 2 (a), (d) and (f) are the surface plots of $m_{e f f} / m_{s t}$ in the global view, Figs. 2 (b), (e) and (g) are the two-dimensional plots of $m_{\text {eff }} / m_{s t}$ at section $\omega / \omega_{4}=3.0$, and Fig. 2 (c) is the two-dimensional plot of $m_{\text {eff }} / m_{s t}$ at section $\omega / \omega_{2}=3.0$. As shown in Figs. 2 (a) - (c), there are four negative effective mass frequency regions. Two of these band gaps are on the $\omega / \omega_{4}$ axis and the other two are on the $\omega / \omega_{2}$ axis. As $\tau_{2}$ and $\tau_{3}$ increase from 0.01 to 1.0 , the second band gap on the $\omega / \omega_{2}$ axis vanishes, and the amplitude of $m_{e f f} / m_{s t}$ at the first resonant frequency is reduced. The main reason of this phenomenon is that the production of negative effective mass is based on the local resonance motions of oscillators. With the increase of damping coefficients, the motions of resonators are mitigated.

From Figs. 2 (h) - (j), there are four band gaps in the metadamping surface plots, and the value of $c_{\text {eff }}$ is always positive. Its amplitude is significantly affected by the local resonance frequencies and the damping coefficients. From Fig. 2 (g), at the resonance frequencies, the effective metadamping coefficient $c_{\text {eff }}$ is sufficiently improved. In Figs. 2 (h) - (l), high $\tau_{2}$ and $\tau_{3}$ reduce the amplitude of $c_{\text {eff }}$ at the resonance frequencies and improve the value of $c_{\text {eff }}$ in other frequency regions. The width of the second band gap on the $\omega / \omega_{2}$ axis is enhanced with the increase of damping coefficients. However, the excessive high damping causes the disappearance of the second band gap. Furthermore, through comparing the cases of $\tau_{2}=0.01 \tau_{3}=$ $0.01, \tau_{2}=1.0 \tau_{3}=0.01$ and $\tau_{2}=0.01 \tau_{3}=1.0$ in Figs. $2(\mathrm{~h}),(\mathrm{j})$ and (l), it is found that the influence of $\tau_{3}$ on the value of $c_{\text {eff }}$ is more significantly than that of $\tau_{2}$. 


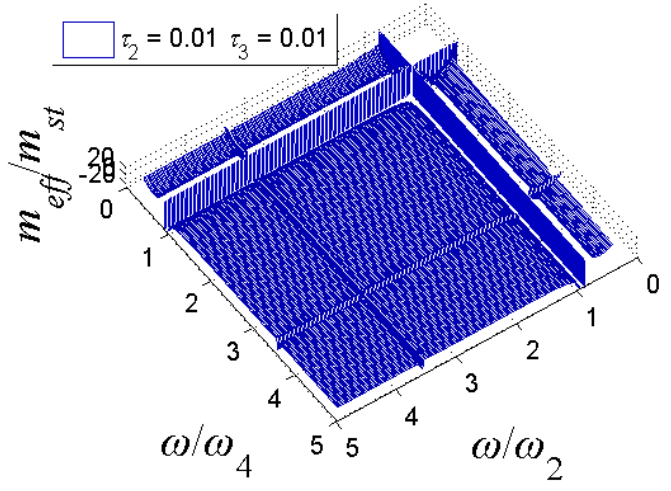

(a)

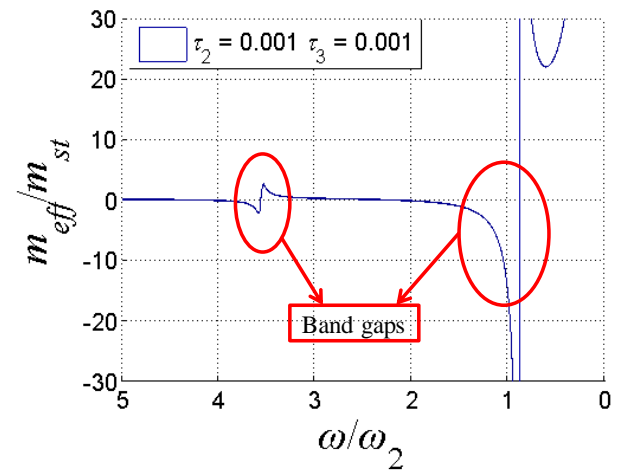

(b)

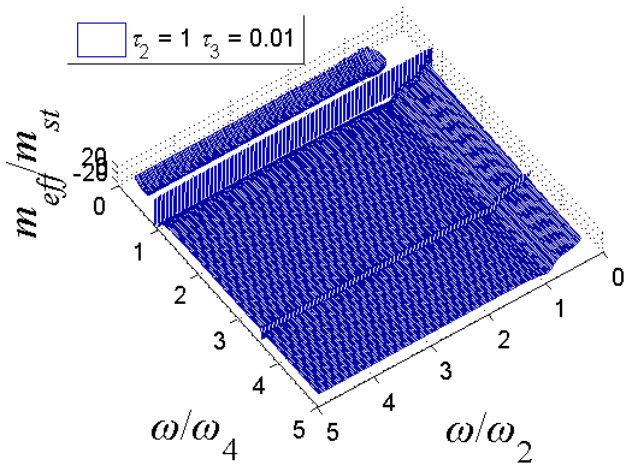

(d)

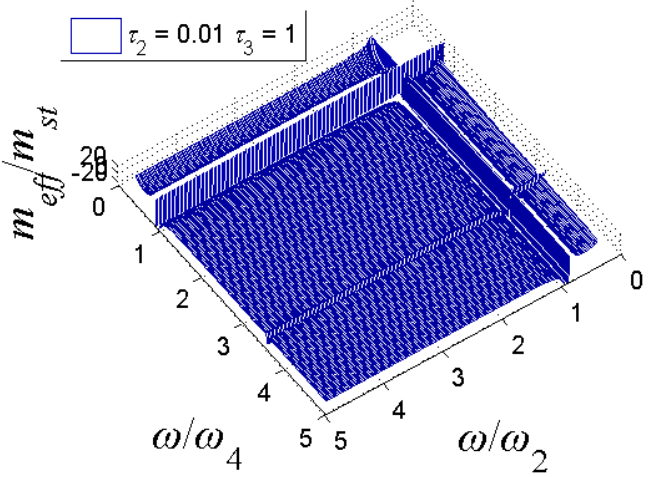

(f)

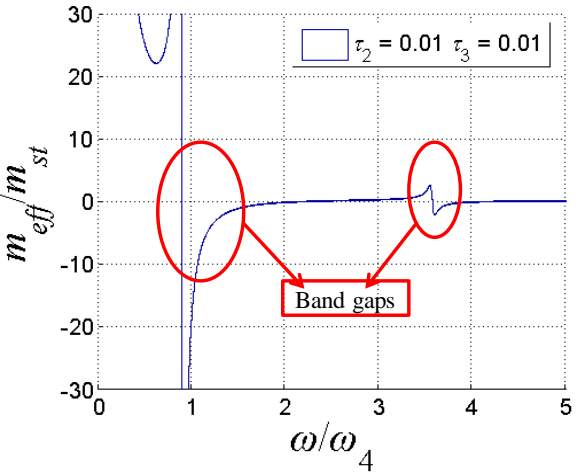

(c)

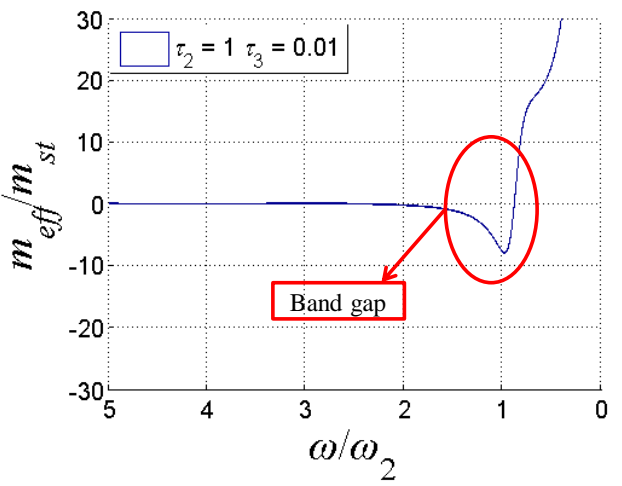

(e)

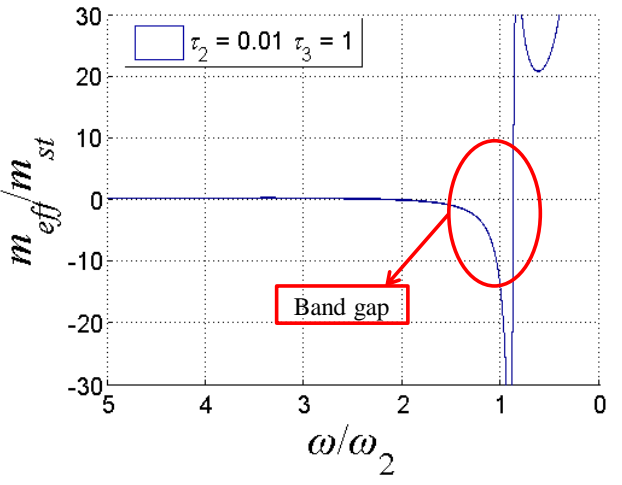

(g) 


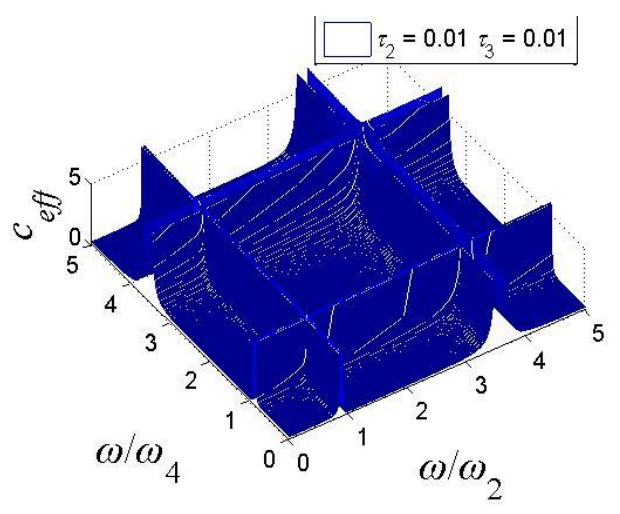

(h)

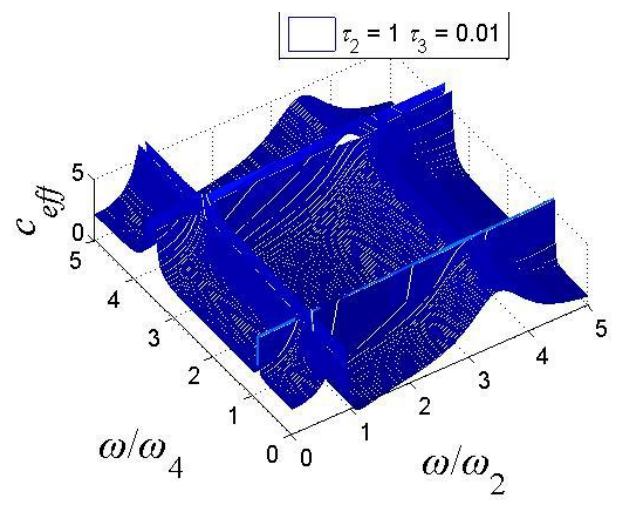

(j)

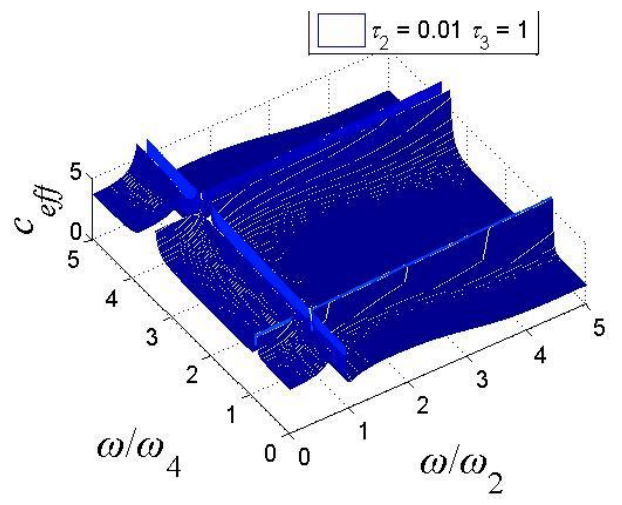

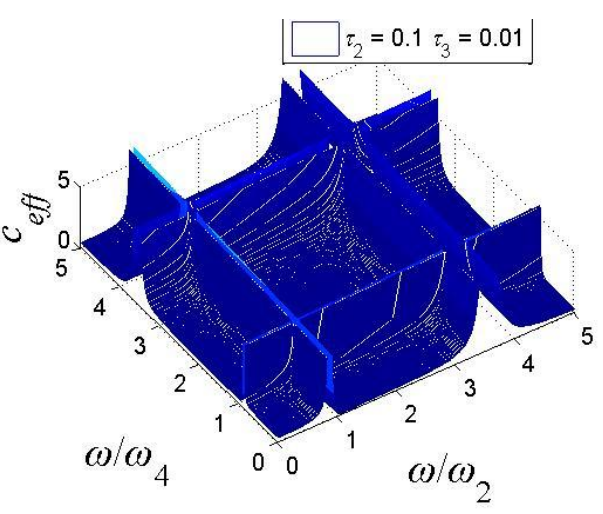

(i)

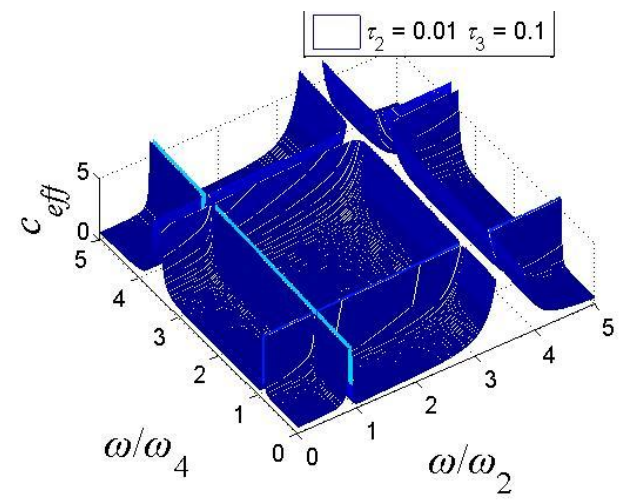

$(\mathrm{k})$

(1)

Fig. 2. (a) Surface plot of dimensionless effective mass against dimensionless frequencies with $\tau_{2}=0.01, \tau_{3}=0.01$ in global view. (b) Plot of $m_{\text {eff }} / m_{s t}$ at section $\omega / \omega_{4}=$ 3.0 with parameters $\tau_{2}=0.01$ and $\tau_{3}=0.01$. (c) Plot of $m_{\text {eff }} / m_{s t}$ at section $\omega / \omega_{2}=3.0$ with parameters $\tau_{2}=0.01$ and $\tau_{3}=0.01$. (d) $\tau_{2}=1.0, \tau_{3}=0.01$ in global view. (e) $\tau_{2}=1.0, \tau_{3}=0.01$ at section $\omega / \omega_{4}=3.0$. (f) $\tau_{2}=0.01, \tau_{3}=1.0$ in global view. (g) $\tau_{2}=0.01, \tau_{3}=1.0$ at section $\omega / \omega_{4}=3.0$. (h) $3 \mathrm{D}$ graph of effective metadamping coefficient against dimensionless frequencies with $\tau_{2}=0.01, \tau_{3}=0.01$ in global view. (i) $\tau_{2}=0.1, \tau_{3}=0.01$ in global view. (j) $\tau_{2}=1.0, \tau_{3}=0.01$ in global view. (k) $\tau_{2}=0.01, \tau_{3}=0.1$ in global view. (l) $\tau_{2}=0.01, \tau_{3}=1.0$ in global view.

The real and imaginary parts of the motion ratios of inner mass $m_{2}$ to outer mass 
$m_{1}$ and middle $m_{3}$ to outer mass $m_{1}$ are given in Fig. 3. In Fig. 3 (a), there are three band gap regions for $\operatorname{Re}\left(u_{2} / u_{1}\right) . u_{2}$ is out of phase with $u_{1}$ at the frequency regions lower than the first resonant frequency, lower than the third resonant frequency and larger than the second resonant frequency. When $\tau_{2}$ is increased from 0.01 to 1 , the amplitude of $\operatorname{Re}\left(u_{2} / u_{1}\right)$ at the second and third resonant frequencies is dramatically decreased, and the value of $\operatorname{Re}\left(u_{2} / u_{1}\right)$ at the first resonant frequency is increased. Similar to $\tau_{2}$, a high $\tau_{3}$ improves the value of $\operatorname{Re}\left(u_{2} / u_{1}\right)$ at the first resonant frequency, and decreases $\operatorname{Re}\left(u_{2} / u_{1}\right)$ at the second and third resonant frequencies. The main reason for the general decrease of the motion ratio $u_{2} / u_{1}$ is that high damping reduces the motions of oscillators. Fig. 3 (b) shows the imaginary part of $u_{2} / u_{1} \cdot \operatorname{Im}\left(u_{2} / u_{1}\right)$ is significantly increased at the resonance frequency regions. With the increase of $\tau_{2}$ and $\tau_{3}$, the second and third sharp peaks of $\operatorname{Im}\left(u_{2} / u_{1}\right)$ decrease, while the first sharp peak increases.

Fig. 3 (c) shows the plots of $\operatorname{Re}\left(u_{3} / u_{1}\right)$ against $\omega / \omega_{2}$. From Fig. 3 (c), $u_{3}$ and $u_{1}$ are in phase at the frequency regions lower than the first resonant frequency and larger than the second resonant frequency. When the frequency is between the first and the second resonant frequencies, $u_{3}$ is always out of phase with $u_{1}$. Similar to $\operatorname{Re}\left(u_{2} / u_{1}\right)$, at the local resonance frequencies, the increase of $\tau_{2}$ and $\tau_{3}$ decreases the amplitude of $\operatorname{Re}\left(u_{3} / u_{1}\right)$. Fig. 3 (d) illustrates the imaginary part of $u_{3} / u_{1}$, and the value of $\operatorname{Im}\left(u_{3} / u_{1}\right)$ is also greatly influenced by the resonance frequencies and the damping coefficients. At the resonance frequencies, $\operatorname{Im}\left(u_{3} / u_{1}\right)$ tends to be very large, and high damping decrease the peak values of $\operatorname{Im}\left(u_{3} / u_{1}\right)$.

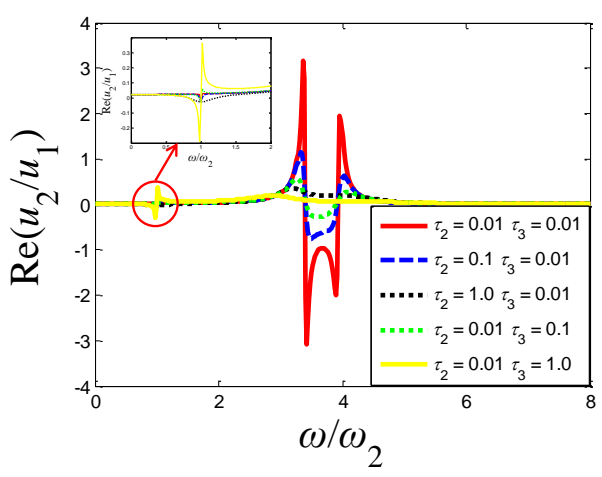

(a)

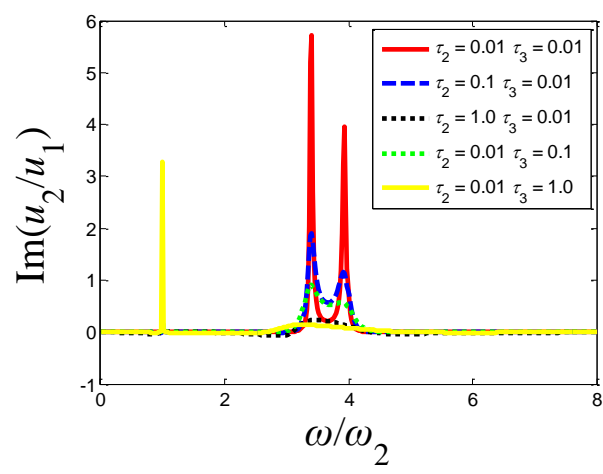

(b) 


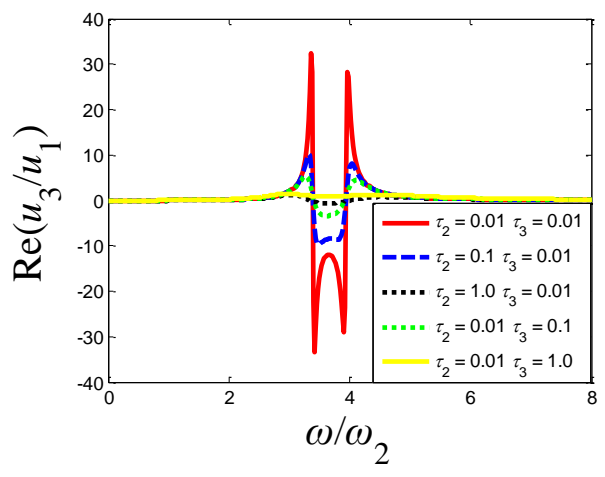

(c)

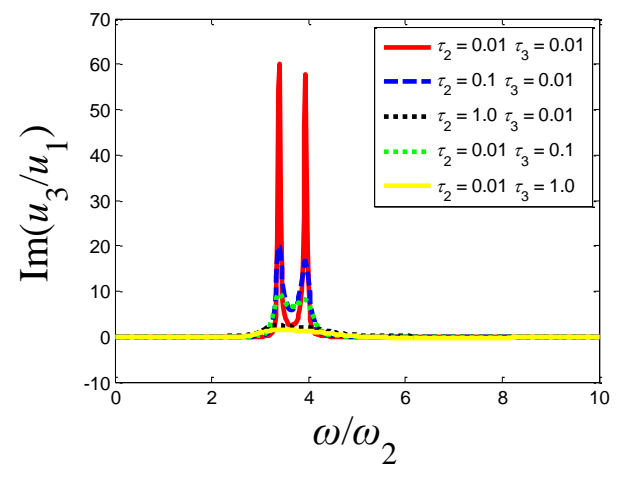

(d)

Fig. 3. (a) Plots of real part of $u_{2} / u_{1}$. (b) Plots of imaginary part of $u_{2} / u_{1}$. (c) Plots of real part of $u_{3} / u_{1}$. (d) Plots of imaginary part of $u_{3} / u_{1}$.

\subsection{MT multi-resonators microstructure}
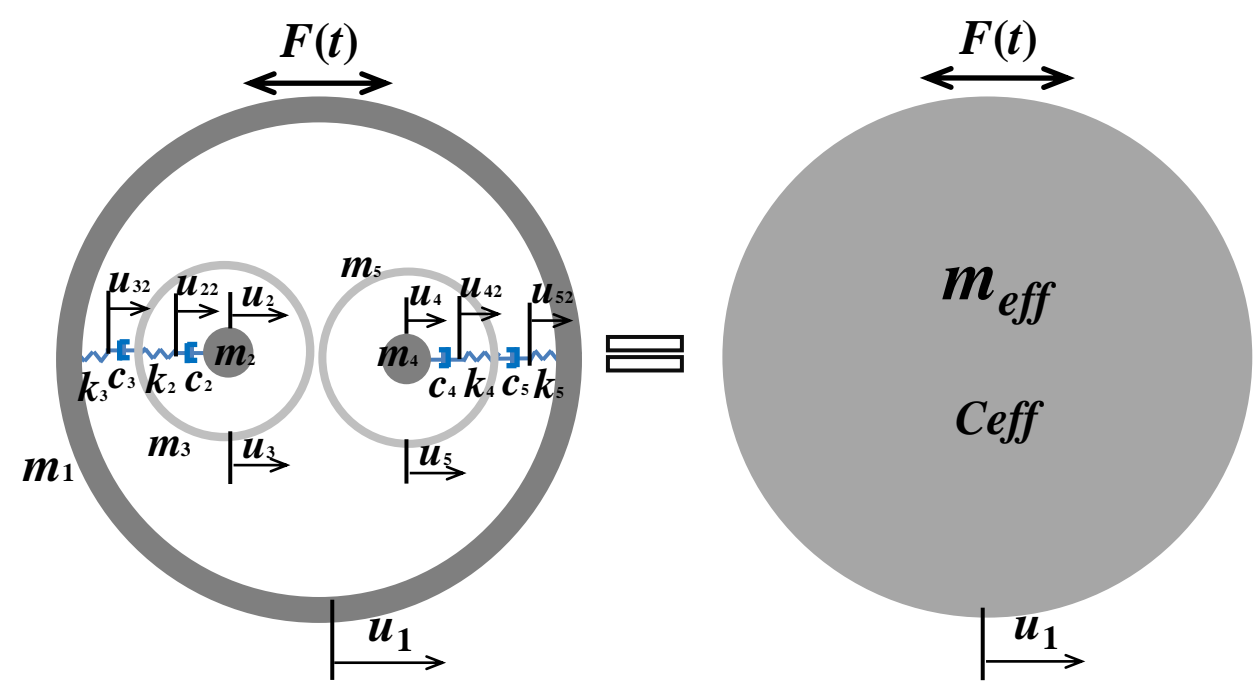

Fig. 4. MT multi-resonators microstructure

The MT multi-resonators microstructure, which consists of four dashpots, four springs and five oscillators, is shown in Fig. 4. Compared with KVT microstructure, MT has additional degrees of freedom at the interfacing points of dashpots and springs, which are $u_{22}, u_{32}, u_{42}$ and $u_{52}$. Similar to KVT, the governing equations of MT microstructure are acquired as follows.

$$
\begin{aligned}
& m_{2} \ddot{u}_{2}=c_{2}\left(\dot{u}_{22}-\dot{u}_{2}\right) \\
& m_{3} \ddot{u}_{3}=k_{2}\left(u_{22}-u_{3}\right)+c_{3}\left(\dot{u}_{32}-\dot{u}_{3}\right) \\
& m_{1} \ddot{u}_{1}=F+k_{3}\left(u_{32}-u_{1}\right)+k_{5}\left(u_{52}-u_{1}\right)
\end{aligned}
$$


$m_{4} \ddot{u}_{4}=c_{4}\left(\dot{u}_{42}-\dot{u}_{4}\right)$

$m_{5} \ddot{u}_{5}=k_{4}\left(u_{42}-u_{5}\right)+c_{5}\left(\dot{u}_{52}-\dot{u}_{5}\right)$

$0=k_{3}\left(u_{1}-u_{32}\right)+c_{3}\left(\dot{u}_{3}-\dot{u}_{32}\right)$

$0=k_{2}\left(u_{3}-u_{22}\right)+c_{2}\left(\dot{u}_{2}-\dot{u}_{22}\right)$

$0=k_{5}\left(u_{1}-u_{52}\right)+c_{5}\left(\dot{u}_{5}-\dot{u}_{52}\right)$

$0=k_{4}\left(u_{5}-u_{42}\right)+c_{4}\left(\dot{u}_{4}-\dot{u}_{42}\right)$

Then, the following equation of this system can be acquired.

$\left[\begin{array}{lll}A_{11} & A_{12} & A_{13} \\ A_{21} & A_{22} & A_{23} \\ A_{31} & A_{32} & A_{33} \\ A_{41} & A_{42} & A_{43} \\ A_{51} & A_{52} & A_{53} \\ A_{61} & A_{62} & A_{63} \\ A_{71} & A_{72} & A_{73} \\ A_{81} & A_{82} & A_{83} \\ A_{91} & A_{92} & A_{93}\end{array}\right]\left\{\begin{array}{l}\omega^{2} \\ \omega \\ 1\end{array}\right\}=\left\{\begin{array}{l}0 \\ 0 \\ 0 \\ 0 \\ 0 \\ 0 \\ 0 \\ 0 \\ 0\end{array}\right\}$

in which

$$
\begin{aligned}
& A_{11}=m_{2} \hat{u}_{2}, A_{21}=m_{3} \hat{u}_{3}, A_{31}=m_{1} \hat{u}_{1}, A_{41}=m_{4} \hat{u}_{4}, A_{51}=m_{5} \hat{u}_{5}, A_{61}=0, A_{71}=0, A_{81}=0, \\
& A_{91}=0, A_{12}=-i \tau_{2} \sqrt{k_{2} m_{2}}\left(\hat{u}_{22}-\widehat{u}_{2}\right), A_{22}=-i \tau_{3} \sqrt{k_{3} m_{3}}\left(\widehat{u}_{32}-\widehat{u}_{3}\right), A_{32}=0, \\
& A_{42}=-i \tau_{4} \sqrt{k_{4} m_{4}}\left(\widehat{u}_{42}-\widehat{u}_{4}\right), A_{52}=-i \tau_{5} \sqrt{k_{5} m_{5}}\left(\widehat{u}_{52}-\widehat{u}_{5}\right), A_{62}=-i \tau_{3} \sqrt{k_{3} m_{3}}\left(\widehat{u}_{3}-\widehat{u}_{32}\right) \text {, } \\
& A_{72}=-i \tau_{2} \sqrt{k_{2} m_{2}}\left(\widehat{u}_{2}-\widehat{u}_{22}\right), A_{82}=-i \tau_{5} \sqrt{k_{5} m_{5}}\left(\widehat{u}_{5}-\widehat{u}_{52}\right), A_{92}=-i \tau_{4} \sqrt{k_{4} m_{4}}\left(\widehat{u}_{4}-\widehat{u}_{42}\right), \\
& A_{13}=0, A_{23}=k_{2}\left(\hat{u}_{22}-\widehat{u}_{3}\right), A_{33}=k_{3}\left(\hat{u}_{32}-\widehat{u}_{1}\right)+k_{5}\left(\hat{u}_{52}-\widehat{u}_{1}\right)+F, \\
& A_{43}=0, A_{53}=k_{4}\left(\hat{u}_{42}-\widehat{u}_{5}\right), A_{63}=k_{3}\left(\widehat{u}_{1}-\widehat{u}_{32}\right), A_{73}=k_{2}\left(\widehat{u}_{3}-\widehat{u}_{22}\right), \\
& A_{83}=k_{5}\left(\widehat{u}_{1}-\widehat{u}_{52}\right), A_{93}=k_{4}\left(\widehat{u}_{5}-\widehat{u}_{42}\right) .
\end{aligned}
$$


Fig. 5 shows the surface plots of $m_{\text {eff }} / m_{s t}$ and $c_{\text {eff }}$ of MT multi-resonator microstructure. The parameter values of spring stiffness and mass ratios of MT model are the same as KVT model, and the dimensionless damping coefficients of $\tau_{4}$ and $\tau_{5}$ are set as 20.0. As shown in Fig. 5 (a), when the damping coefficients are set as $\tau_{2}=1.0$ and $\tau_{3}=1.0$, there is no band gap on the $\omega / \omega_{2}$ axis. From Figs. 5 (a) - (c), as $\tau_{2}$ and $\tau_{3}$ increase from 1.0 to 20.0 , two band gaps gradually appear. This is because the spring and the damping are linear in series in the MT multi-resonator microstructure, and the stiffness of this system depends on the smaller value of the spring stiffness and damping stiffness. The damper with high value acts as a rigid connection between the resonator and the spring, which can improve the resonance motions of oscillators. Figs. 5 (b) and (c) show that the increase of $\tau_{2}$ and $\tau_{3}$ improves the amplitudes of these two band gaps on the $\omega / \omega_{2}$ axis, while reduces their width. In addition, $\tau_{2}$ and $\tau_{3}$ have different effects on the band gaps. From cases $\tau_{2}=20.0 \quad \tau_{3}=20.0, \tau_{2}=50.0 \quad \tau_{3}=20$ and $\tau_{2}=20.0 \tau_{3}=50$ in Figs. 5 (c) - (e), it can be seen that the increase of $\tau_{2}$ significantly enhances the amplitude of the second band gap, while a high $\tau_{3}$ improves the first band gap more.

As shown in Fig. 5 (f), when the damping parameters are $\tau_{2}=1.0$ and $\tau_{3}=1.0$, there is no band gap on the $\omega / \omega_{2}$ axis for the surface plot of $c_{\text {eff. }}$ From Figs. 5 (g) and (h), as $\tau_{2}$ and $\tau_{3}$ increase from 5.0 to 20.0 , the value of $c_{e f f}$ at the resonance frequencies improves significantly, and its band gap width decreases. Furthermore, from Figs. 5 (h) - (j), the first and second band gaps on the $\omega / \omega_{2}$ axis are promoted with the increases of $\tau_{2}$, and a large $\tau_{3}$ enhances the amplitude of first band gap obviously. It is worth noting that high $c_{\text {eff }}$ value and broad $c_{\text {eff }}$ band gap mean great dissipation effect of DAMs. Large negative effective mass frequency range and high amplitude of $m_{e f f} / m_{s t}$ imply great attenuation effect. Therefore, a reasonable damping coefficient is beneficial to both the attenuation and dissipation effects of MT multi-resonators microstructure. 


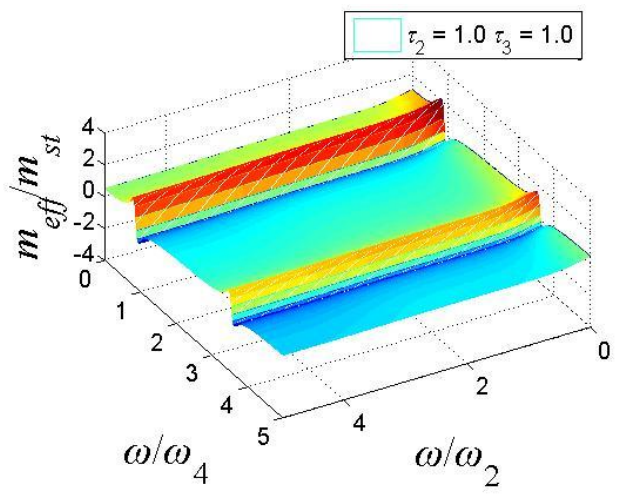

(a)

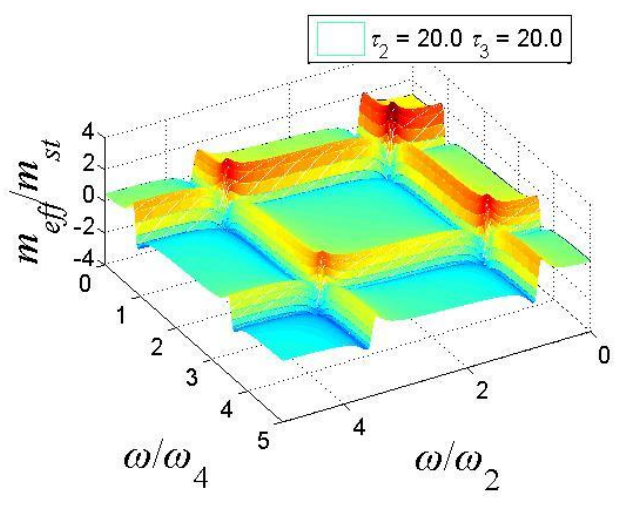

(c)

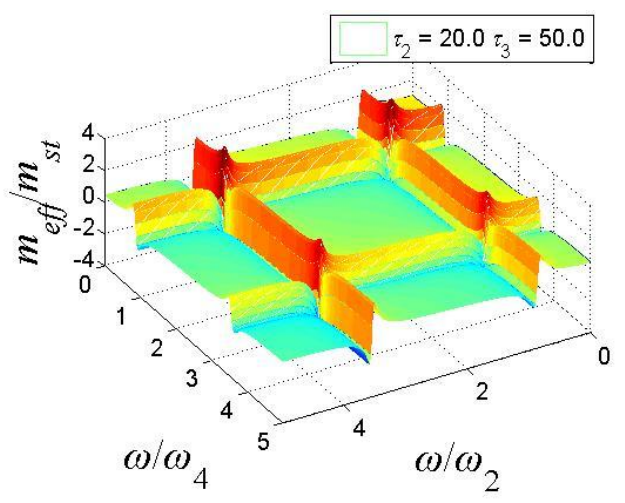

(e)

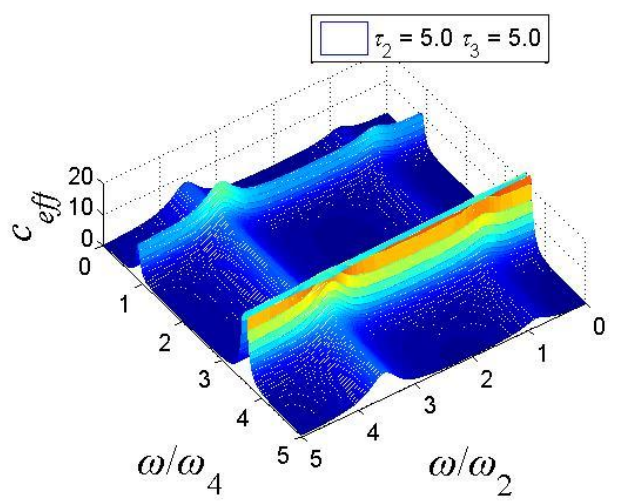

(g)

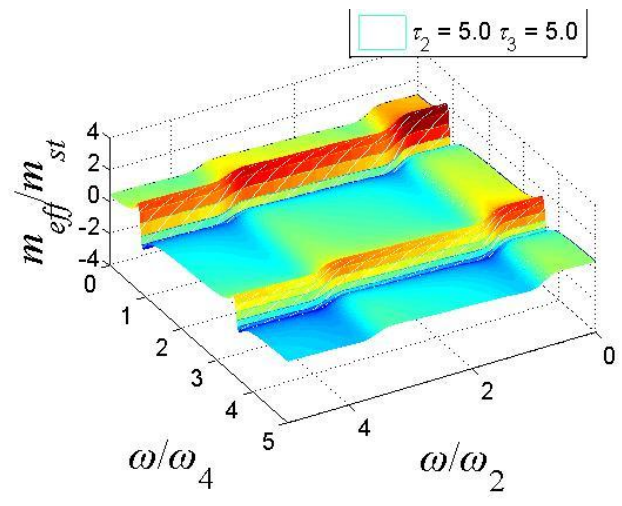

(b)

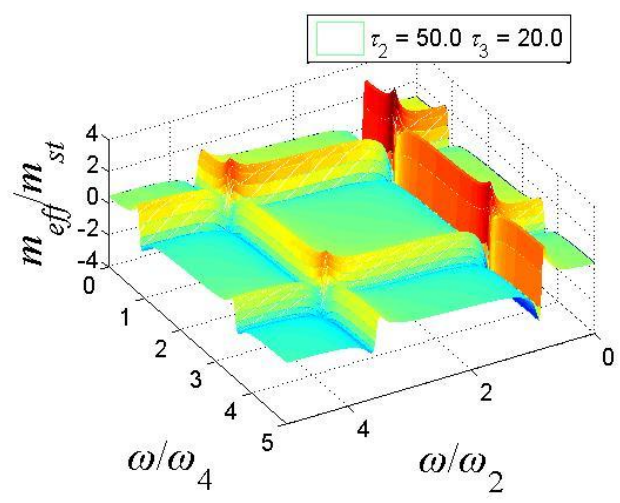

(d)

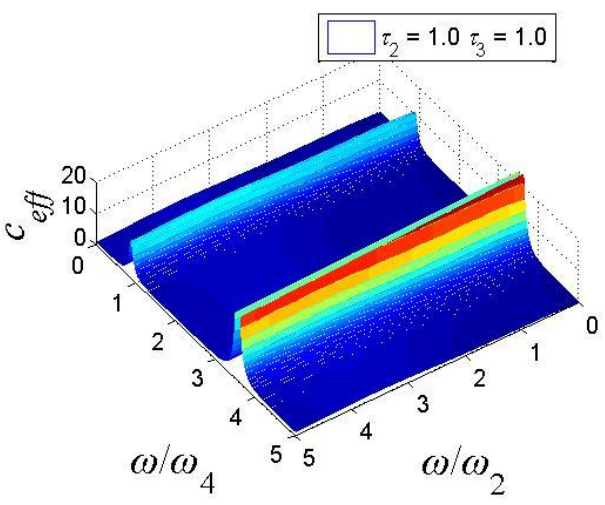

(f)

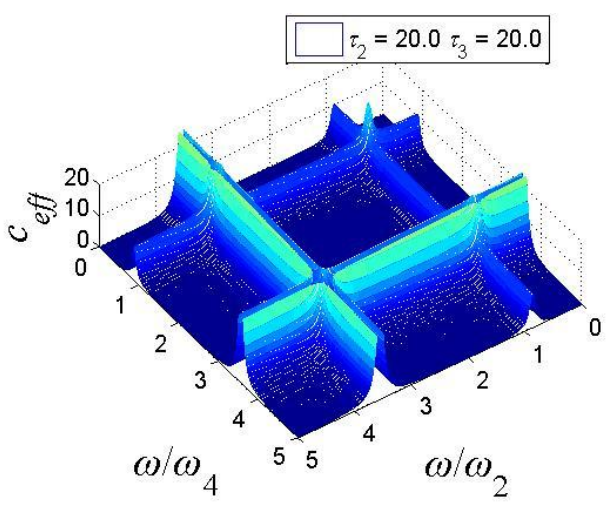

(h) 


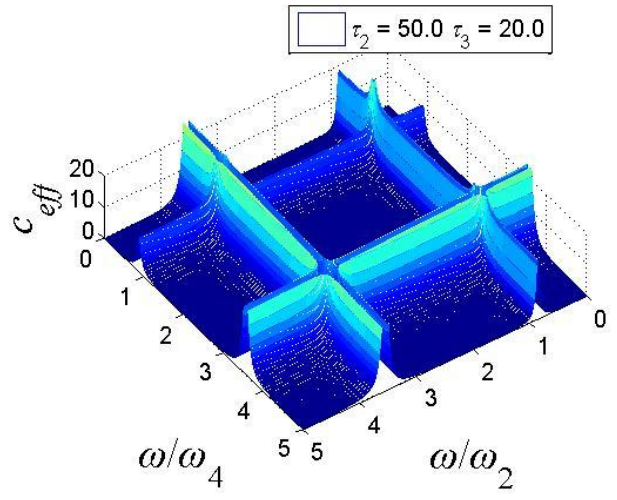

(i)

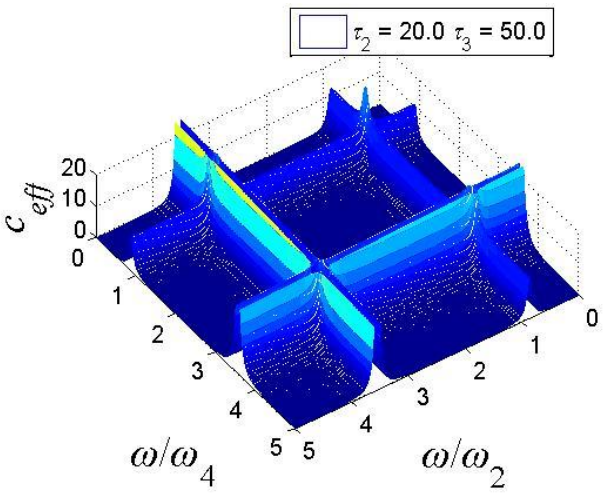

(j)

Fig. 5. (a) Surface plot of dimensionless effective mass against dimensionless frequencies with $\tau_{2}=1.0, \tau_{3}=1.0$. (b) $\tau_{2}=5.0, \tau_{3}=5.0$. (c) $\tau_{2}=20.0, \tau_{3}=20.0$. (d) $\tau_{2}=50.0$, $\tau_{3}=20.0$. (e) $\tau_{2}=20.0, \tau_{3}=50.0$. (f) $3 \mathrm{D}$ graph of effective metadamping coefficients against dimensionless frequencies with $\tau_{2}=1.0, \tau_{3}=1.0$. (g) $\tau_{2}=5.0, \tau_{3}=5.0$. (h) $\tau_{2}=20.0$, $\tau_{3}=20.0$. (i) $\tau_{2}=50.0, \tau_{3}=20.0$. (j) $\tau_{2}=20.0, \tau_{3}=50.0$.

The motion ratios of MT oscillators are shown in Fig. 6, and the movements of resonators are significantly affected by the resonant frequencies and the damping coefficients. In Fig. 6 (a), there are two band gaps, and $u_{2}$ is out of phase with $u_{1}$ at the frequency ranges lower than the first resonant frequency. At the frequency regions lower than the second resonant frequency and larger the first resonant frequency, the motions of $u_{2}$ and $u_{1}$ are in phase with each other first and then become out of phase. When the frequency spectrum is larger than the second resonant frequency, $u_{2}$ is always in phase with $u_{1}$. In addition, the peak value of $\operatorname{Re}\left(u_{2} / u_{1}\right)$ at the resonant frequencies increases with the increase of $\tau_{2}$ and $\tau_{3}$. Based on the parameter cases of $\tau_{2}$ $=20.0 \tau_{3}=20.0, \tau_{2}=50.0 \tau_{3}=20.0$ and $\tau_{2}=20.0 \tau_{3}=50.0$, a high $\tau_{2}$ significantly enhances the oscillator motions at the first resonant frequency, and the increase of $\tau_{3}$ greatly improves the vibrator movements at the second resonant frequency.

In Fig. 6 (c), there are also two band gaps for $\operatorname{Re}\left(u_{3} / u_{1}\right) . u_{3}$ is in phase with $u_{1}$ at the frequency regions lower than the first resonant frequency. When the frequency regions are between the first and second resonant frequencies, the motions of $m_{3}$ and $m_{1}$ are out of phase at first and then become in phase. At the frequency ranges which are larger than the second resonant frequency, $u_{3}$ is always out of phase with $u_{1}$. In addition, with the increase of $\tau_{2}$ and $\tau_{3}$, the motion of $m_{3}$ is more intense, and large $\tau_{2}$ 
and $\tau_{3}$ significantly enhance the value of $\operatorname{Re}\left(u_{3} / u_{1}\right)$ at the second resonant frequency. In Figs. 6 (b) and (d), there are two peak sharps in the $\operatorname{Im}\left(u_{2} / u_{1}\right)$ and $\operatorname{Im}\left(u_{3} / u_{1}\right)$ plots. Similar to $\operatorname{Re}\left(u_{2} / u_{1}\right)$ and $\operatorname{Re}\left(u_{3} / u_{1}\right)$, large $\tau_{2}$ and $\tau_{3}$ enhance the peak values of $\operatorname{Im}\left(u_{2} / u_{1}\right)$ and $\operatorname{Im}\left(u_{3} / u_{1}\right)$.

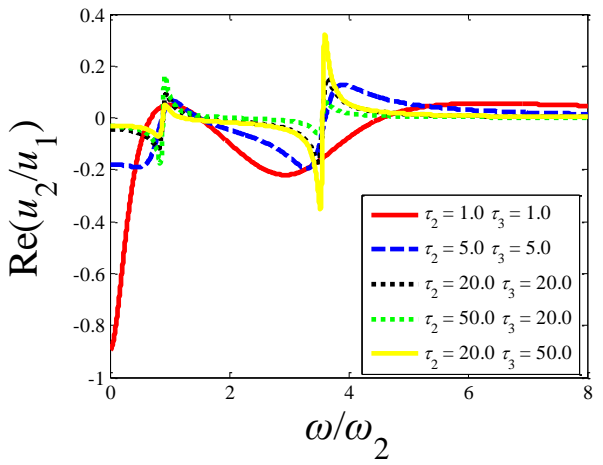

(a)

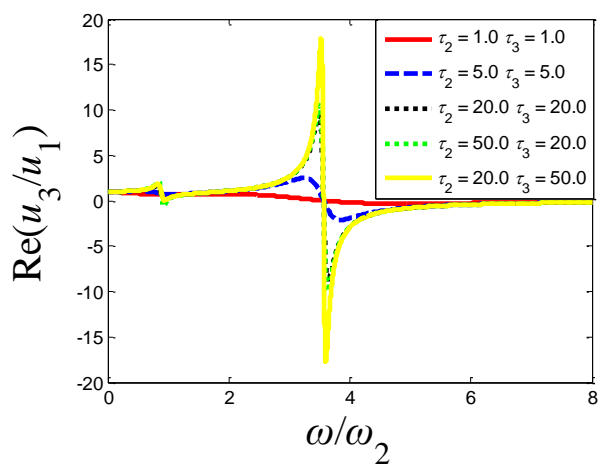

(c)

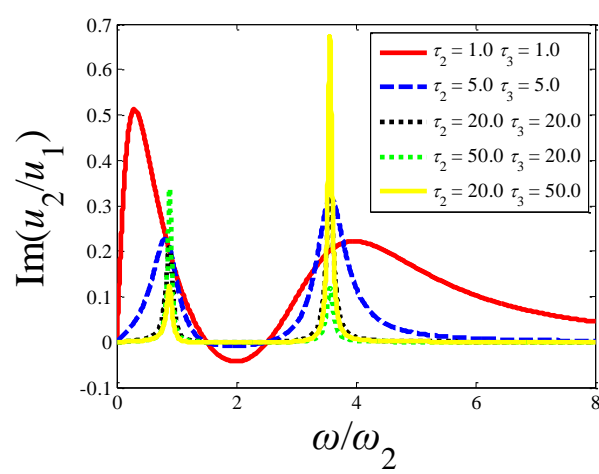

(b)

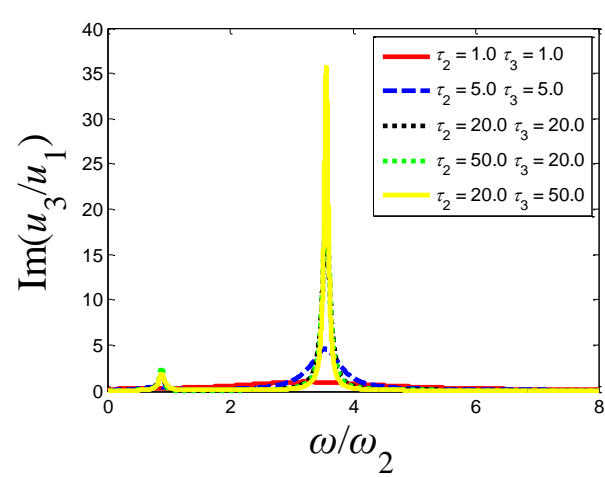

(d)

Fig. 6. (a) Plot of $\operatorname{Re}\left(u_{2} / u_{1}\right)$ against $\omega / \omega_{2}$. (b) Plots of $\operatorname{Im}\left(u_{2} / u_{1}\right)$ against $\omega / \omega_{2}$. (c) Graph of $\operatorname{Re}\left(u_{3} / u_{1}\right)$ against $\omega / \omega_{2}$. (d) Graph of $\operatorname{Im}\left(u_{3} / u_{1}\right)$ against $\omega / \omega_{2}$. 

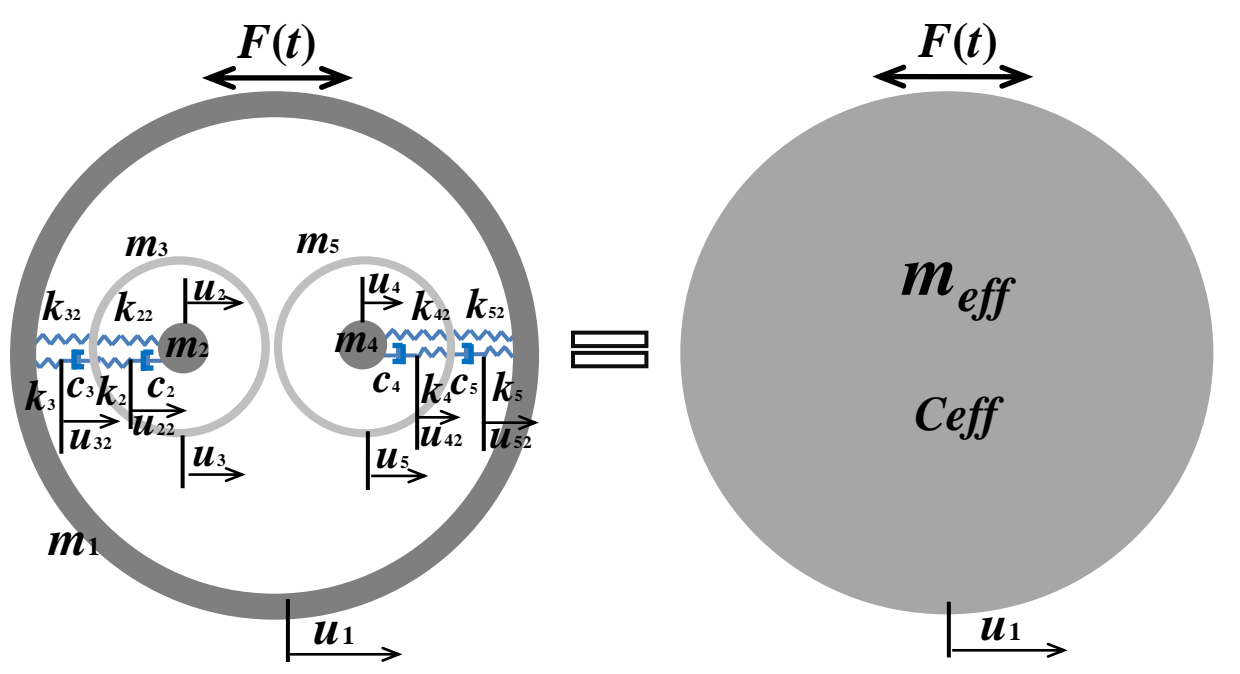

Fig. 7. ZT multi-resonators microstructure

As shown in Fig. 7, the configuration features of the ZT multi-resonator microstructure are combined by the KVT and MT microstructures. Compared with $\mathrm{KVT}$, four additional tunable stiffness parameters are introduced to represent the springs that are connected to the dashpots and the resonators. Analogously, the equations of these freedoms in ZT microstructure are written as follows.

$$
\begin{aligned}
& m_{2} \ddot{u}_{2}=k_{22}\left(u_{3}-u_{2}\right)+c_{2}\left(\dot{u}_{22}-\dot{u}_{2}\right) \\
& m_{3} \ddot{u}_{3}=k_{2}\left(u_{22}-u_{3}\right)+c_{3}\left(\dot{u}_{32}-\dot{u}_{3}\right)+k_{22}\left(u_{2}-u_{3}\right)+k_{32}\left(u_{1}-u_{3}\right) \\
& m_{1} \ddot{u}_{1}=F+k_{3}\left(u_{32}-u_{1}\right)+k_{32}\left(u_{3}-u_{1}\right)+k_{5}\left(u_{52}-u_{1}\right)+k_{52}\left(u_{5}-u_{1}\right) \\
& m_{4} \ddot{u}_{4}=k_{42}\left(u_{5}-u_{4}\right)+c_{4}\left(\dot{u}_{42}-\dot{u}_{4}\right) \\
& m_{5} \ddot{u}_{5}=k_{4}\left(u_{42}-u_{5}\right)+c_{5}\left(\dot{u}_{52}-\dot{u}_{5}\right)++k_{42}\left(u_{4}-u_{5}\right)+k_{52}\left(u_{1}-u_{5}\right) \\
& 0=k_{3}\left(u_{1}-u_{32}\right)+c_{3}\left(\dot{u}_{3}-\dot{u}_{32}\right) \\
& 0=k_{2}\left(u_{3}-u_{22}\right)+c_{2}\left(\dot{u}_{2}-\dot{u}_{22}\right) \\
& 0=k_{5}\left(u_{1}-u_{52}\right)+c_{5}\left(\dot{u}_{5}-\dot{u}_{52}\right) \\
& 0=k_{4}\left(u_{5}-u_{42}\right)+c_{4}\left(\dot{u}_{4}-\dot{u}_{42}\right)
\end{aligned}
$$


We obtain the following Eq. (9) by simplifying Eq. (8).

$$
\left[\begin{array}{lll}
A_{11} & A_{12} & A_{13} \\
A_{21} & A_{22} & A_{23} \\
A_{31} & A_{32} & A_{33} \\
A_{41} & A_{42} & A_{43} \\
A_{51} & A_{52} & A_{53} \\
A_{61} & A_{62} & A_{63} \\
A_{71} & A_{72} & A_{73} \\
A_{81} & A_{82} & A_{83} \\
A_{91} & A_{92} & A_{93}
\end{array}\right]\left\{\begin{array}{l}
\omega^{2} \\
\omega \\
1
\end{array}\right\}=\left\{\begin{array}{l}
0 \\
0 \\
0 \\
0 \\
0 \\
0 \\
0 \\
0 \\
0
\end{array}\right\}
$$

in which

$$
\begin{aligned}
& A_{11}=m_{2} \hat{u}_{2}, A_{21}=m_{3} \hat{u}_{3}, A_{31}=m_{1} \hat{u}_{1}, A_{41}=m_{4} \hat{u}_{4}, A_{51}=m_{5} \widehat{u}_{5}, A_{61}=0, A_{71}=0, A_{81}=0, \\
& A_{91}=0, A_{12}=-i \tau_{2} \sqrt{k_{2} m_{2}}\left(\widehat{u}_{22}-\widehat{u}_{2}\right), A_{22}=-i \tau_{3} \sqrt{k_{3} m_{3}}\left(\widehat{u}_{32}-\widehat{u}_{3}\right), A_{32}=0, \\
& A_{42}=-i \tau_{4} \sqrt{k_{4} m_{4}}\left(\widehat{u}_{42}-\widehat{u}_{4}\right), A_{52}=-i \tau_{5} \sqrt{k_{5} m_{5}}\left(\widehat{u}_{52}-\widehat{u}_{5}\right), A_{62}=-i \tau_{3} \sqrt{k_{3} m_{3}}\left(\widehat{u}_{3}-\widehat{u}_{32}\right), \\
& A_{72}=-i \tau_{2} \sqrt{k_{2} m_{2}}\left(\widehat{u}_{2}-\widehat{u}_{22}\right), A_{82}=-i \tau_{5} \sqrt{k_{5} m_{5}}\left(\widehat{u}_{5}-\widehat{u}_{52}\right), A_{92}=-i \tau_{4} \sqrt{k_{4} m_{4}}\left(\widehat{u}_{4}-\widehat{u}_{42}\right), \\
& A_{13}=k_{22}\left(\widehat{u}_{3}-\widehat{u}_{2}\right), A_{23}=k_{2}\left(\widehat{u}_{22}-\widehat{u}_{3}\right)+k_{22}\left(\hat{u}_{2}-\widehat{u}_{3}\right)+k_{32}\left(\hat{u}_{1}-\widehat{u}_{3}\right), \\
& A_{33}=k_{3}\left(\widehat{u}_{32}-\widehat{u}_{1}\right)+k_{32}\left(\widehat{u}_{3}-\widehat{u}_{1}\right)+k_{5}\left(\widehat{u}_{52}-\widehat{u}_{1}\right)+k_{5}\left(\widehat{u}_{5}-\widehat{u}_{1}\right)+\widehat{F}, \\
& A_{43}=k_{42}\left(\widehat{u}_{5}-\widehat{u}_{4}\right), A_{53}=k_{4}\left(\hat{u}_{42}-\widehat{u}_{5}\right)+k_{42}\left(\hat{u}_{4}-\widehat{u}_{5}\right)+k_{52}\left(\hat{u}_{1}-\widehat{u}_{5}\right), \\
& A_{63}=k_{3}\left(\hat{u}_{1}-\widehat{u}_{32}\right), A_{73}=k_{2}\left(\widehat{u}_{3}-\widehat{u}_{22}\right), A_{83}=k_{5}\left(\widehat{u}_{1}-\widehat{u}_{52}\right), A_{93}=k_{4}\left(\widehat{u}_{5}-\widehat{u}_{42}\right) \text {. }
\end{aligned}
$$

Fig. 8 shows the surface graphs of dimensionless effective mass $m_{\text {eff }} / m_{s t}$ of ZT microstructure against $\omega / \omega_{2}$ and $\omega / \omega_{4}$. In this model, the parameter values of dimensionless damping coefficients $\tau_{2}, \tau_{3}, \tau_{4}$ and $\tau_{5}$, stiffness ratios $k_{2} / k_{3}$ and $k_{4} / k_{5}$, and mass ratios $m_{2} / m_{3}, m_{3} / m_{1}, m_{4} / m_{5}$ and $m_{5} / m_{1}$ are the same as KVT. The additional spring stiffness ratios of $k_{22} / k_{32}$ and $k_{42} / k_{52}$ are the same as $k_{2} / k_{3}$ and $k_{4} / k_{5}$. Similar to KVT and MT, ZT also has four negative effective mass frequency regions and four effective metadamping band gaps.

In Figs. 8 (a) - (e), the second band gaps of $m_{\text {eff }} / m_{s t}$ on the $\omega / \omega_{2}$ and $\omega / \omega_{4}$ axes are small, which implies the resonance motions of the middle masses $m_{3}$ and $m_{5}$ are 
not as intense as the inner masses $m_{2}$ and $m_{4}$. From Figs. 8 (a) - (c), with the increase of $\tau_{2}$, the amplitude of $m_{e f f} / m_{s t}$ at the two resonant frequencies of $\omega / \omega_{2}$ axis and the second resonant frequency of $\omega / \omega_{4}$ axis are significantly reduced. Especially for the first band gap of $\omega / \omega_{2}$ axis, the decline is dramatic. As shown in Figs. 8 (a), (d) and (e), a high $\tau_{3}$ decreases the amplitude of $m_{\text {eff }} / m_{s t}$ at the resonance frequencies, and the second band gap on the $\omega / \omega_{2}$ axis vanishes as $\tau_{3}$ increases from 0.01 to 1.0 .

The conditions of effective metadamping coefficient $c_{\text {eff }}$ are similar to those of $m_{\text {eff }} / m_{s t}$. In Figs. 8 (f) - (j), the increase of $\tau_{2}$ and $\tau_{3}$ decreases the value of $c_{\text {eff }}$ at the second resonant frequency on the $\omega / \omega_{2}$ axis, while it enhances the width of the second band gap. Through the combination of metadamping and local resonance, the dissipation and attenuation effects of DAMs with the optimal damping coefficients can be sufficiently improved.

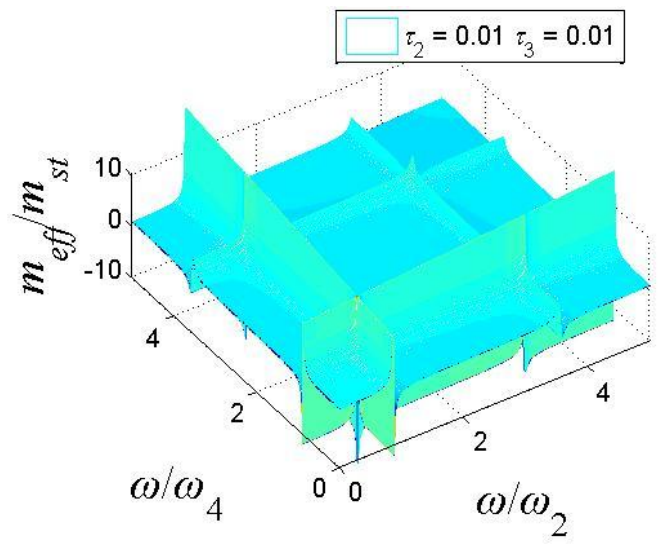

(a)

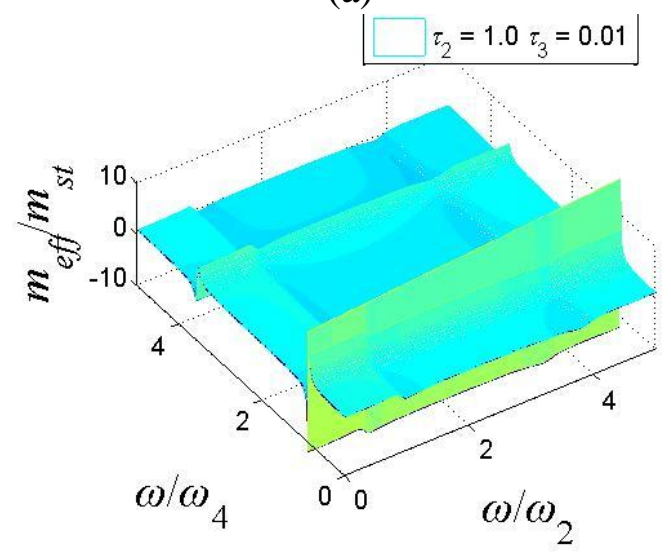

(c)

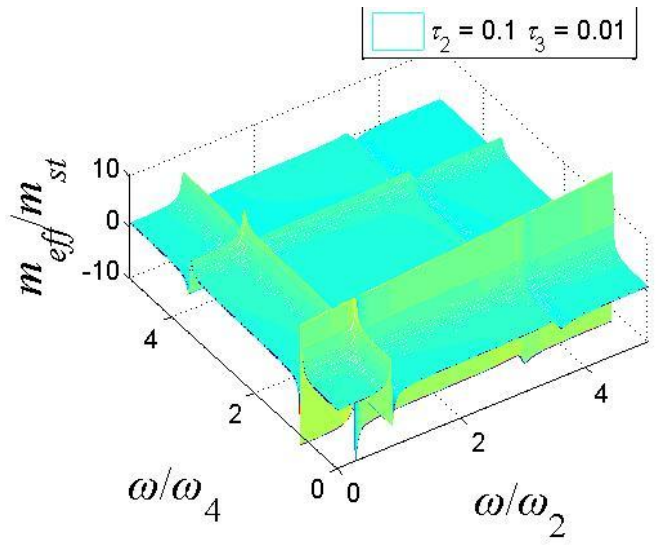

(b)

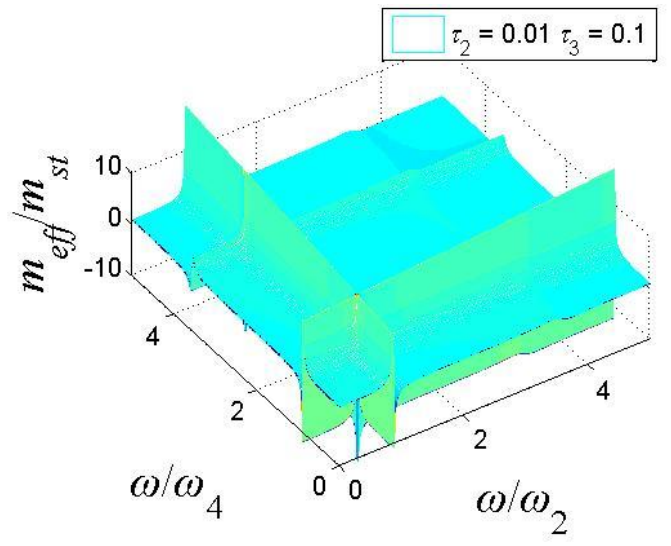

(d) 


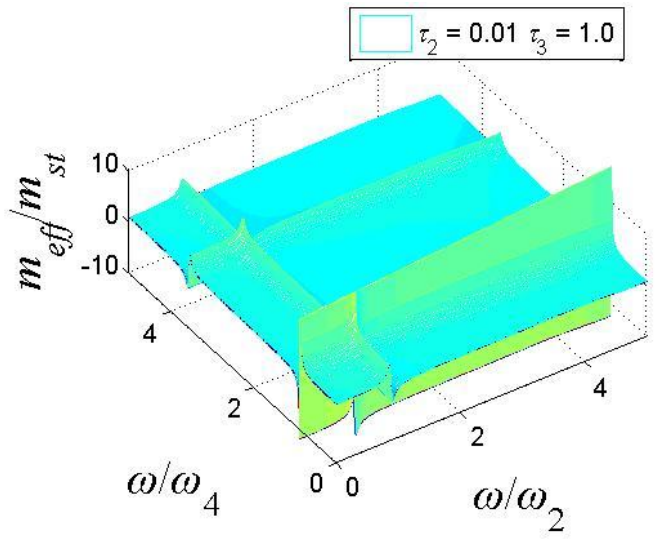

(e)

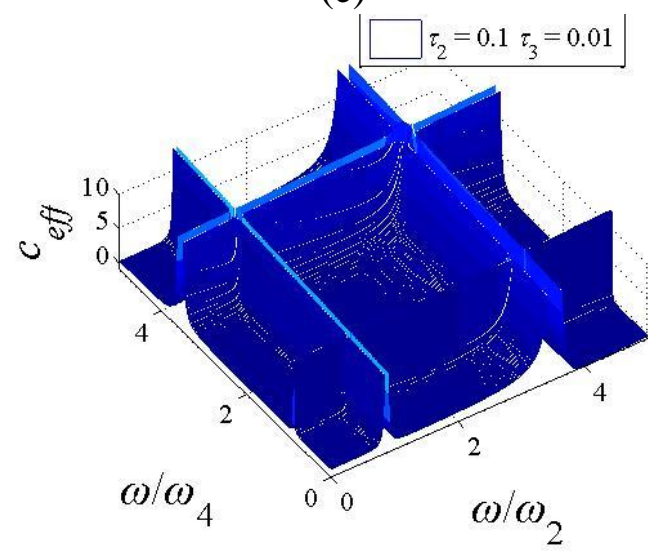

(g)

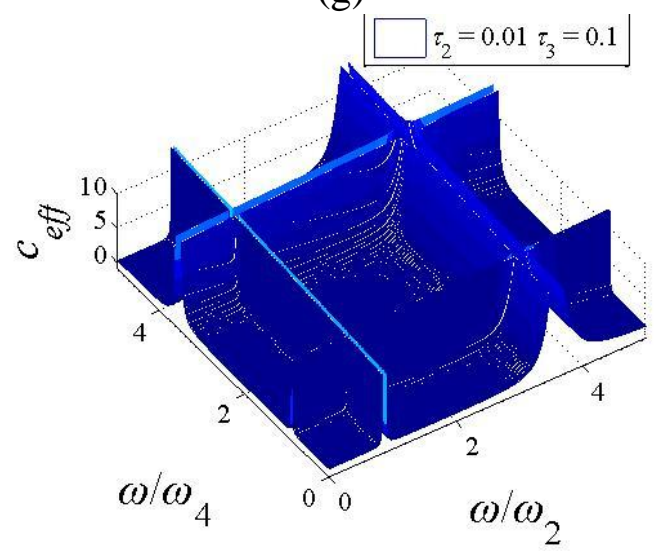

(i)

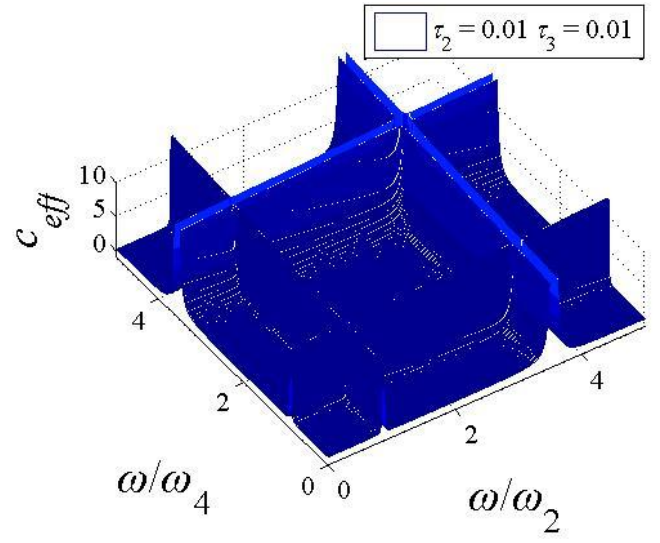

(f)

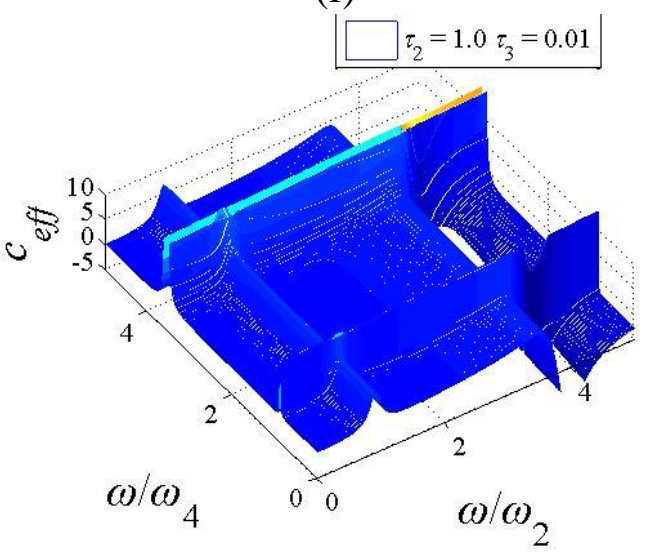

(h)

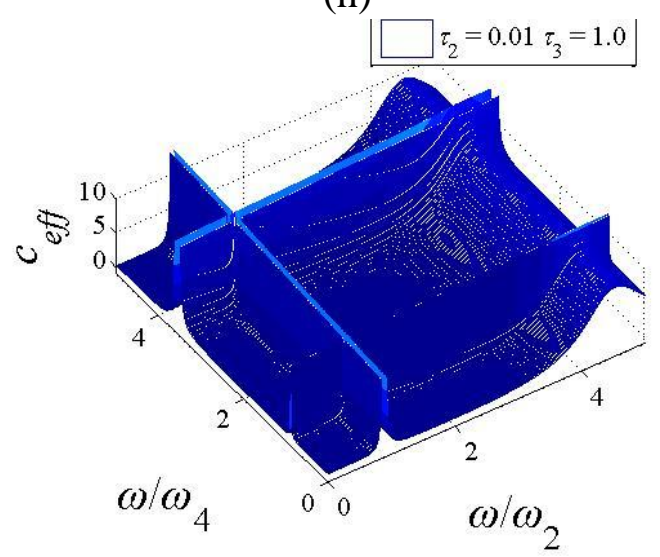

(j)

Fig. 8. (a) Surface plot of dimensionless effective mass against non-dimensional frequencies with $\tau_{2}=0.01, \tau_{3}=0.01$ (b) $\tau_{2}=0.1, \tau_{3}=0.01$. (c) $\tau_{2}=1.0, \tau_{3}=0.01$. (d) $\tau_{2}=0.01$, $\tau_{3}=0.1$. (e) $\tau_{2}=0.01, \tau_{3}=1.0$. (f) Surface plot of effective metadamping coefficient against non-dimensional frequencies with $\tau_{2}=0.01, \tau_{3}=0.01$. (g) $\tau_{2}=0.1, \tau_{3}=0.01$. (h) $\tau_{2}=1.0, \tau_{3}=0.01$. (i) $\tau_{2}=0.01, \tau_{3}=0.1$. (j) $\tau_{2}=0.01, \tau_{3}=1.0$.

Fig. 9 shows the movement ratios of $m_{2}$ to $m_{1}$ and $m_{3}$ to $m_{1}$. For the real parts of $u_{2} / u_{1}$ and $u_{3} / u_{1}$, there are two band gaps. $u_{2}$ and $u_{3}$ are in phase with $u_{1}$ at the frequency ranges lower than the first resonant frequency. When the frequency increases and occupies the regions between the first and the second resonant 
frequencies, $u_{3}$ is initially out of phase with $u_{1}$ and then gradually becomes in phase with $u_{1}$, while $u_{2}$ is always out of phase with $u_{1}$. After the second resonant frequency, $u_{2}$ is in phase with $u_{1}$, and $u_{3}$ becomes out of phase with $u_{1}$. Furthermore, based on the value of $\operatorname{Re}\left(u_{2} / u_{1}\right)$, the motion of $m_{2}$ at the first resonant frequency is more intense than its movement at the second resonant frequency. In contrast, $m_{3}$ exhibits more intense movement at the second resonant frequency than its resonant motion at the first resonant frequency. In Figs. 9 (a) and (c), with the increase of damping coefficients $\tau_{2}$ and $\tau_{3}$, the motion ratios of $u_{2} / u_{1}$ and $u_{3} / u_{1}$ at the first and second resonant frequencies are obviously reduced, which means the reduction of negative effective mass.

Regarding the imaginary parts, the positive and negative peak values of $\operatorname{Im}\left(u_{2} / u_{1}\right)$ at the first and second resonant frequencies are obtained from Fig. 9 (b). As shown in Fig. 9 (d), there are two band gaps of $\operatorname{Im}\left(u_{3} / u_{1}\right)$ in the frequency ranges. With the increase of $\tau_{2}$ and $\tau_{3}$, the amplitude of $\operatorname{Im}\left(u_{2} / u_{1}\right)$ and $\operatorname{Im}\left(u_{3} / u_{1}\right)$ decreases, while their band gap regions improves.

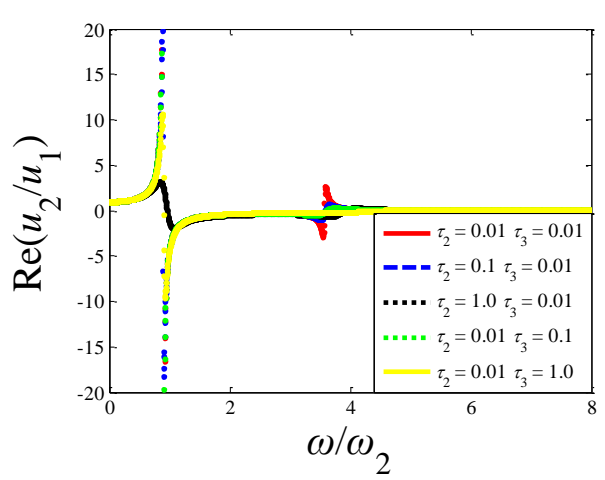

(a)

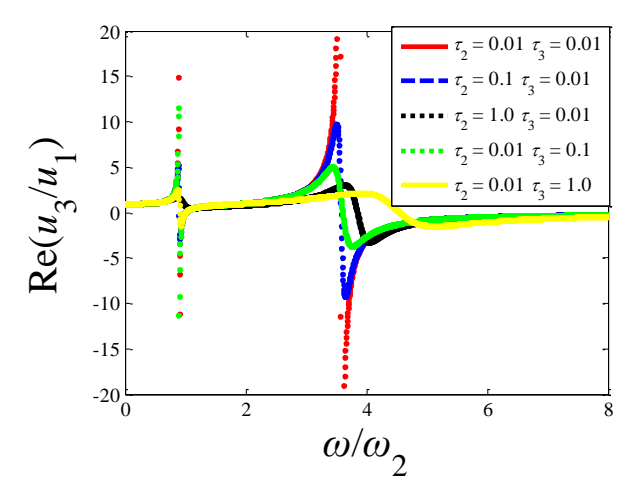

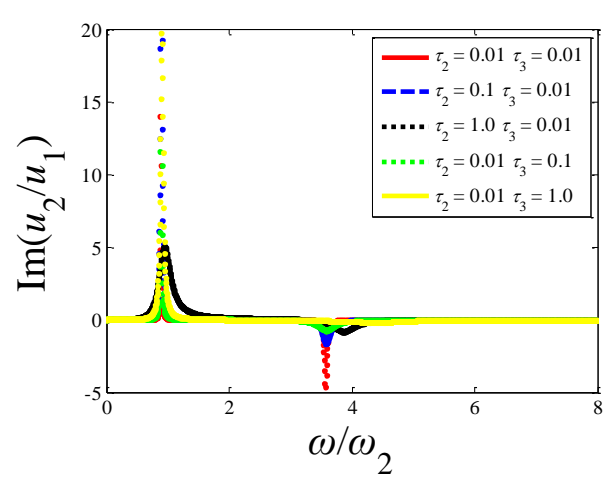

(b)

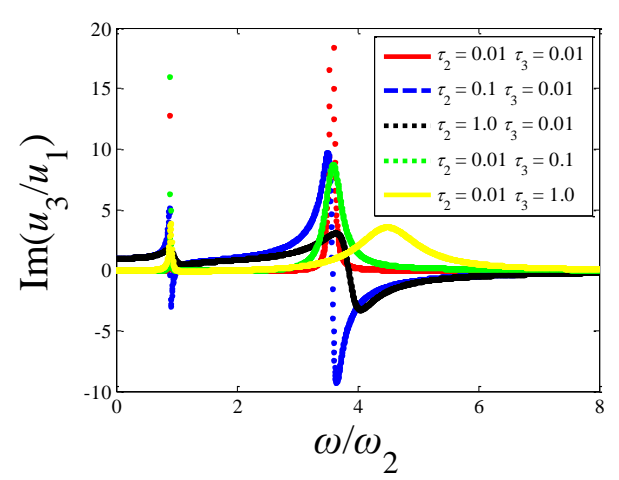


(c)

(d)

Fig. 9. (a) Plots of $\operatorname{Re}\left(u_{2} / u_{1}\right)$ against $\omega / \omega_{2}$. (b) Plots of $\operatorname{Im}\left(u_{2} / u_{1}\right)$ against $\omega / \omega_{2}$. (c) Plots of $\operatorname{Re}\left(u_{3} / u_{1}\right)$ against $\omega / \omega_{2}$. (d) Plots of $\operatorname{Im}\left(u_{3} / u_{1}\right)$ against $\omega / \omega_{2}$.

\section{Impact wave model}

\subsection{Study of the impact wave model}

We further study the attenuation and dissipation effects of these three kinds of DAMs with the impact wave model. A total of 400 unit cells are contained in this impact model, and the unit cells \#101 to \#105 are the damped multi-resonators. The inner masses and outer masses of these unit cells are all set as $0.03 \mathrm{~kg}$. The spring stiffness are $k_{3}=2.665 \mathrm{kN} / \mathrm{m}, k_{2}=0.296 \mathrm{kN} / \mathrm{m}, k_{5}=4.737 \mathrm{kN} / \mathrm{m}$ and $k_{4}=1.184 \mathrm{kN} / \mathrm{m}$ for KVT, MT and ZT microstructures, and the additional spring stiffness for ZT are $k_{32}=2.665 \mathrm{kN} / \mathrm{m}, k_{22}=0.296 \mathrm{kN} / \mathrm{m}, k_{52}=4.737 \mathrm{kN} / \mathrm{m}$ and $k_{42}=1.184 \mathrm{kN} / \mathrm{m}$. The

equation of the impact wave is $F=\widehat{F} e^{-\left(t-t_{0}\right) / t_{d}}\left(t_{0}=0.5 \mathrm{~ms}, t_{d}=0.1 \mathrm{~ms}\right)$, which duration time is $4 \mathrm{~ms}$. The wave is applied to the unit cell \#1. The analysis is carried out with commercial software LSDYNA.

Table 1. Numerical results of these DAMs

\begin{tabular}{cccc}
\hline & Damping $(\mathrm{Nm} / \mathrm{s})$, & Peak velocity $(\mathrm{m} / \mathrm{s})$ & $\begin{array}{c}\text { Peak impact force } \\
(\mathrm{kN})\end{array}$ \\
\cline { 2 - 3 } Original & $c_{2}, c_{3}, c_{4}, c_{5}$ & 0.171 & 0.301 \\
KVT & - & 0.097 & 0.172 \\
MT & $2.25,10.25,10.25,5.25$ & 0.165 & 0.291 \\
ZT & & 0.104 & 0.184 \\
Designed & $4.25,667.42,665.10,3.61$ & 0.087 & 0.153 \\
KVT & & & \\
\hline
\end{tabular}

The velocity and impact force of unit cell \#120 are exhibited as the output responses. Fig. 10 and Table 1 show the results of KVT, MT and ZT DAMs. In Fig. 10 , the damping coefficients of all DAMs are $c_{1}=2.25 \mathrm{Nm} / \mathrm{s}, c_{2}=10.25 \mathrm{Nm} / \mathrm{s}, c_{3}=$ $10.25 \mathrm{Nm} / \mathrm{s}$ and $c_{4}=5.25 \mathrm{Nm} / \mathrm{s}$. Figs. 10 (a) and (c) are the impact force and unit cell velocity response curves. Fig. 10 (b) is the frequency spectrum of impact force curves which are transformed by fast Fourier transform (FFT). 


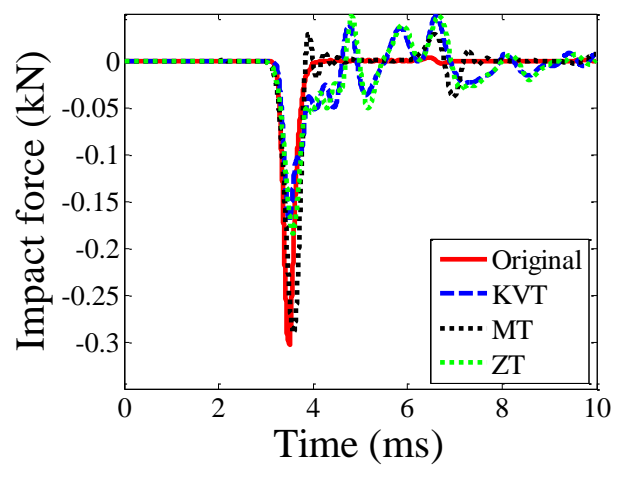

(a)

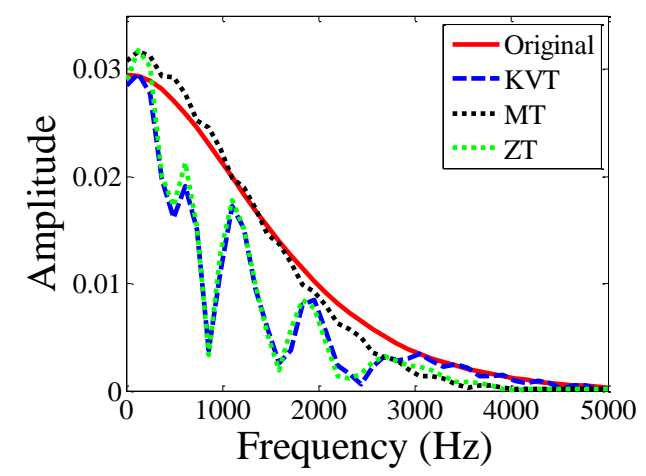

(b)

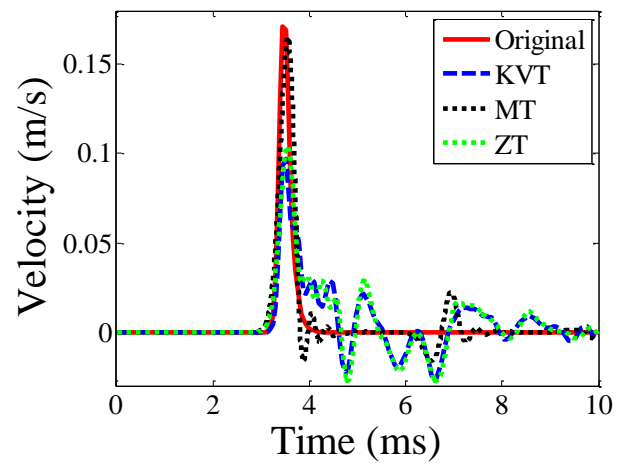

(c)

Fig. 10. (a) Impact force responses of KVT, MT and ZT models. (b) Attenuation of frequency spectrums of KVT, MT and ZT models. (c) Velocity responses of KVT, MT and ZT models.

As shown in Fig. 10 and Table 1, it is clear that the impact force and unit cell velocity behind the DAMs are greatly attenuated. Therefore, DAMs can effectively mitigate the impact wave. The transmissibility of each model is computed by comparing the results of DAM models with the responses of original model. From Figs. 10 (a), (c) and Table 1, it can see that the peak velocities of original, KVT, MT and ZT models are $0.171 \mathrm{~m} / \mathrm{s}, 0.097 \mathrm{~m} / \mathrm{s}, 0.165 \mathrm{~m} / \mathrm{s}$ and $0.104 \mathrm{~m} / \mathrm{s}$. On the other hand, the peak impact forces of these models are $0.301 \mathrm{kN}, 0.172 \mathrm{kN}, 0.291 \mathrm{kN}$ and 0.184 $\mathrm{kN}$. With current damping coefficients, it is clear that the attenuation effects of KVT on the impact force and unit cell velocity are the best among these models, and the performance of ZT is between MT and KVT. These imply that the motions of KVT and ZT oscillators are intense. Much impact energy is stored and absorbed in KVT and ZT damped multi-resonators, which achieve the great attenuation effects. In Fig. 10 (b), both of KVT and ZT have four similar band gaps, while the band gap of MT is 
not obvious. The poor performance of MT is mainly because of the small damping. The small damping coefficients reduce the connection stiffness between the oscillators and alleviate the motion intensity of all vibrators. The broad band gap regions of KVT and ZT prove their high mitigation effects of impact force. These also explain the relationship between frequency spectrum attenuation in the frequency domain and the dynamic load mitigation in the time domain.

\subsection{Maximize the band gap of $K V T$}

It is of interest to obtain the maximum attenuation effects of these DAMs. It is clear that the performance of KVT is great for its smallest peak impact force among these DAMs. Therefore, in this section, we take KVT as an example to study the damping parameters matching to maximize the attenuation effects. The damping coefficients are applied as the design variables. The design space of each variable is from $1 \mathrm{Nm} / \mathrm{s}$ to $1000 \mathrm{Nm} / \mathrm{s}$. The goal is to minimize the peak impact force, and the AT algorithm [55] is applied to do the optimization.

AT was proposed in 2017 by Li et al., which is inspired by the growth law of trees. In AT, the location of the branch represents the design variable, the branch itself is the solution and the branch thickness is the evaluation index of the solution. By simulating the update process of branches and the transfer process of organic matter, the calculation flow of AT is realized. Through the computing of various typical optimization problems, the accuracy and efficiency of the AT algorithm have been proved. Therefore, AT is found to be a very efficient algorithm that can be used to deal with various problems. 


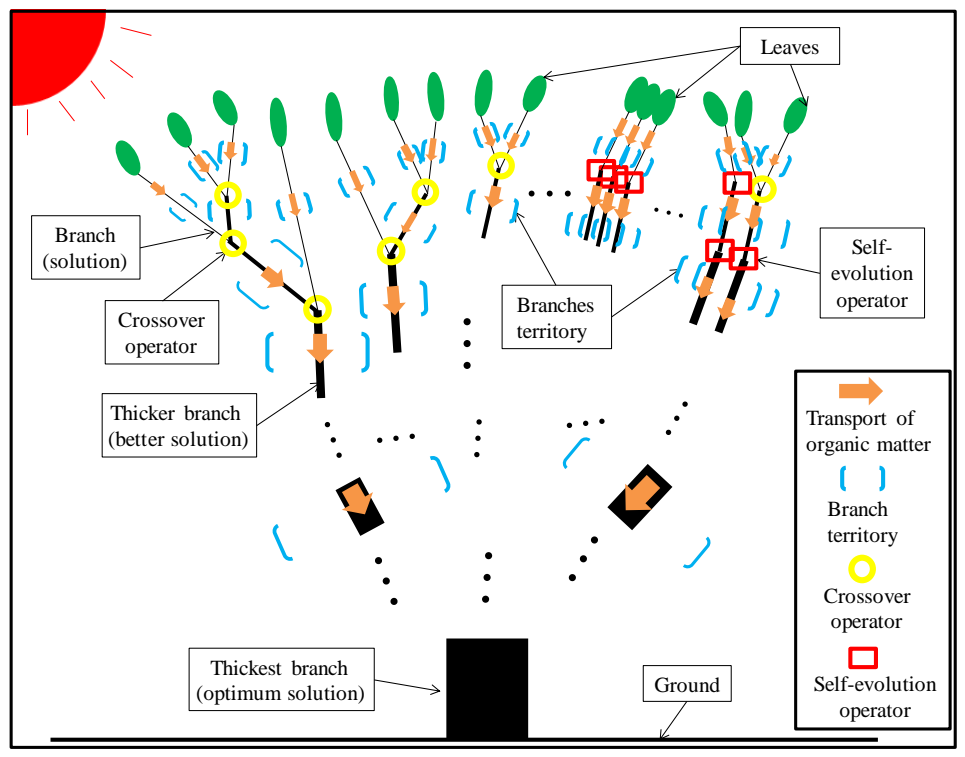

Fig. 11. The growth law of trees

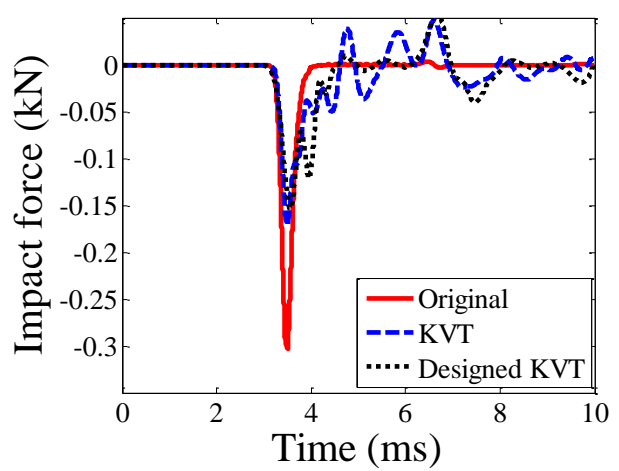

(a)

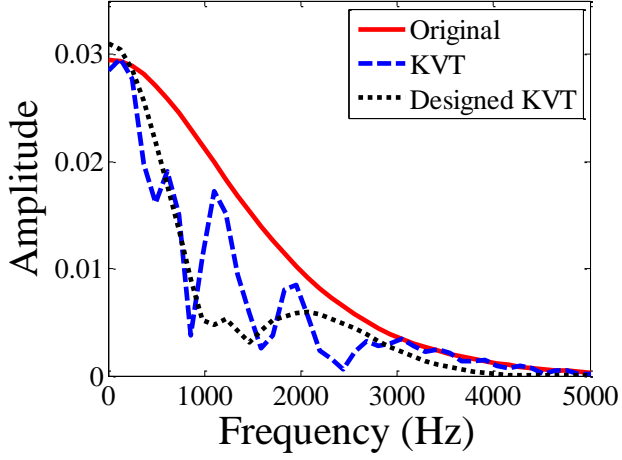

(b)

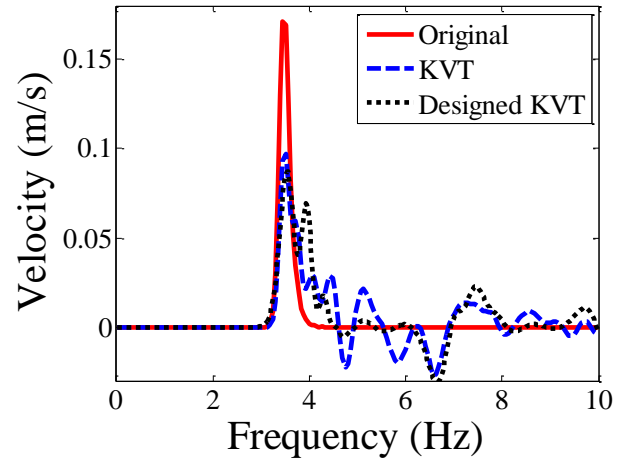

(c)

Fig. 12. (a) Impact force response of KVT and designed KVT. (b) Attenuation of frequency spectrum of KVT and designed KVT. (c) Velocity response of KVT and designed KVT.

The optimization results of KVT model are shown in Fig. 12 and Table. 1. From

Figs. 12 (a) and (c), compared with original KVT model, the peak values of the 
impact force and the velocity of the designed KVT multi-resonator model decrease from $0.172 \mathrm{kN}$ and $0.097 \mathrm{~m} / \mathrm{s}$ to $0.153 \mathrm{kN}$ and $0.087 \mathrm{~m} / \mathrm{s}$. Better mitigation effects are achieved without changing the stiffness of springs and the mass of oscillators. The optimized damping coefficients of $c_{2}, c_{3}, c_{4}$ and $c_{5}$ are $4.25 \mathrm{Nm} / \mathrm{s}, 667.42 \mathrm{Nm} / \mathrm{s}$, $665.10 \mathrm{Nm} / \mathrm{s}$ and 3.61 Nm/s. From Fig. 12 (b), instead of four distinct band gaps, the frequency attenuation regions are integrated into a wide band gap for the designed KVT oscillators. This means the damping can gather the band gaps of the elastic metamaterial together, which is conducive to attenuate the critical frequency regions.

\section{Collision analysis of the dissipative three-dimensional sandwich panel structure}

\subsection{Study of the dissipative three-dimensional sandwich panel structure}

This case is about the collision analysis of a three-dimensional sandwich panel structure. As illustrated in Fig. 13 (a), a rigid circular tube, which mass is $0.16 \mathrm{~kg}$, vertically impacts a three-dimensional panel structure with the initial speed of 13.8 $\mathrm{m} / \mathrm{s}$. The whole panel structure is clamped and fully constrained by two clamping structure at both ends of the plate. This dissipative plate structure is made up of the multi-resonator DAMs and the three-dimensional sandwich panel structure. As shown in Fig. 13 (b), the three-dimensional sandwich panel structure is composed of a cover plate, a base plate and a decahedral honeycomb structure, and their thicknesses are all $1.5 \mathrm{~mm}$. The material of each component is aluminum alloy 6060-T6, and Fig. 13(c) shows its effective stress and effective strain curve. The nonlinear impact analysis is applied with LSDYNA, and the duration time of the collision analysis is $20 \mathrm{~ms}$. Fig. 13 (d) shows that one decahedral honeycomb structure contains one damped KVT multi-resonators unit cell, and these three kinds of dissipative oscillators are included in this sandwich panel structure in turn. The size of one decahedral honeycomb structure is shown in Fig. 13 (e). The rigid masses of $m_{2}, m_{3}, m_{4}$ and $m_{5}$ in these DAMs are all set as $0.005 \mathrm{~kg}$. For KVT, MT and ZT microstructures, the spring 
stiffness of $k_{2}, k_{3}, k_{4}$ and $k_{5}$ are $10.0 \mathrm{~N} / \mathrm{mm}, 31.4 \mathrm{~N} / \mathrm{mm}, 7.9 \mathrm{~N} / \mathrm{mm}$ and $45.2 \mathrm{~N} / \mathrm{mm}$, the value of additional stiffness of $k_{22}, k_{32}, k_{42}$ and $k_{52}$ in ZT are the same as $k_{2}, k_{3}, k_{4}$ and $k_{5}$, respectively. As shown in Table 2, four groups of damping parameters are selected to study the impact responses of this dissipative panel. The damping coefficients in group 4 are the largest among these groups, and the coefficients in group 3 are greater than those of groups 1 and 2. Group 1 has the smallest damping coefficients. Then, how the increase of these damping parameters affects the performances of KVT, MT and ZT oscillators is studied in this section. The results of these dissipative metamaterial panel models contain the dynamic load, the impact energy, the structural deformation and the structural stress.
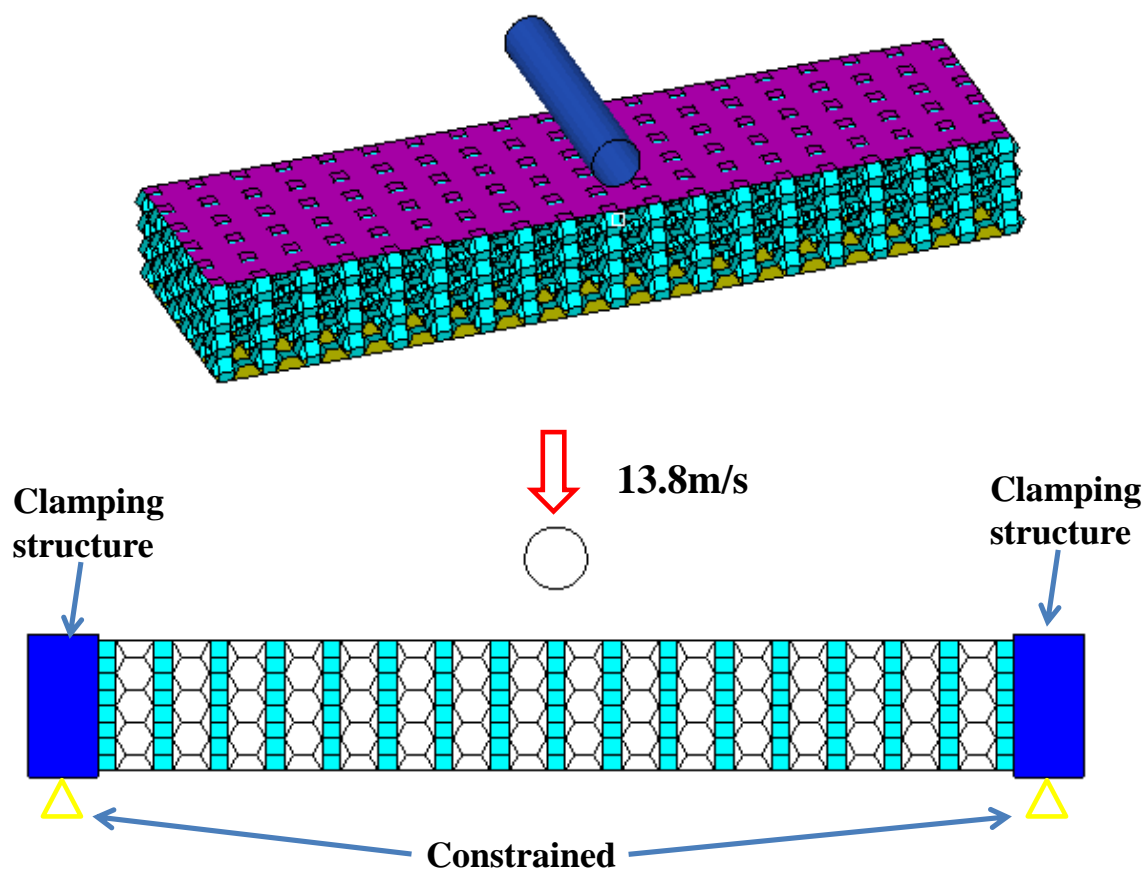

(a) 


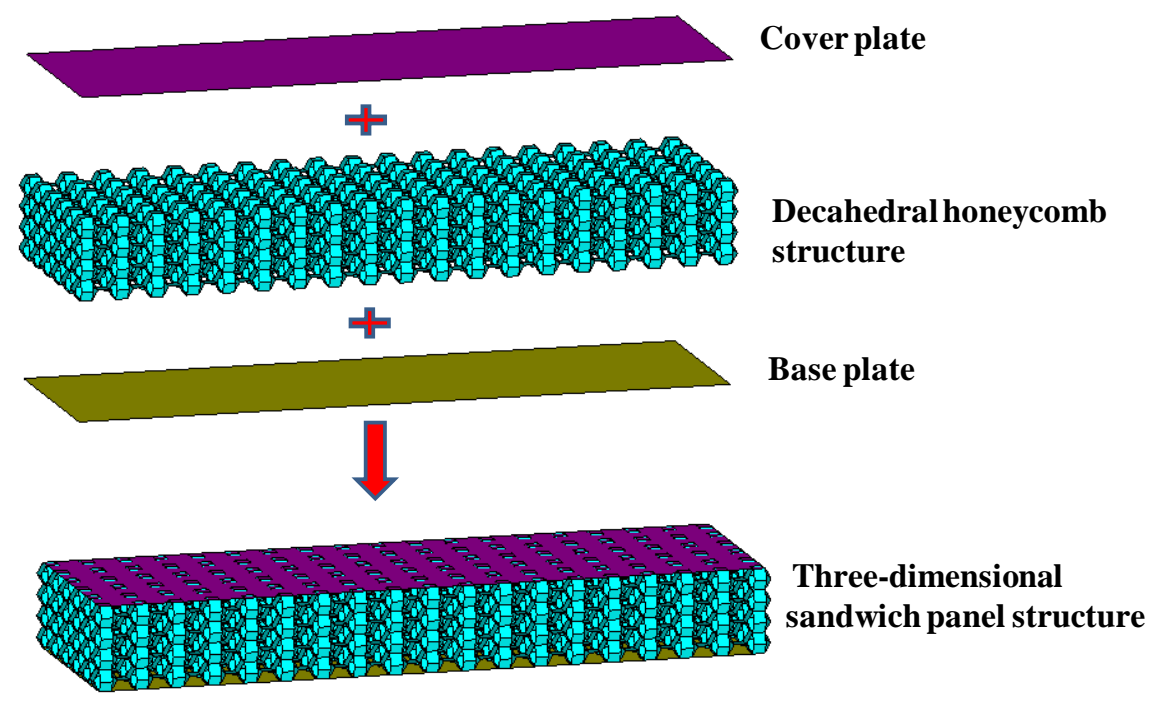

(b)

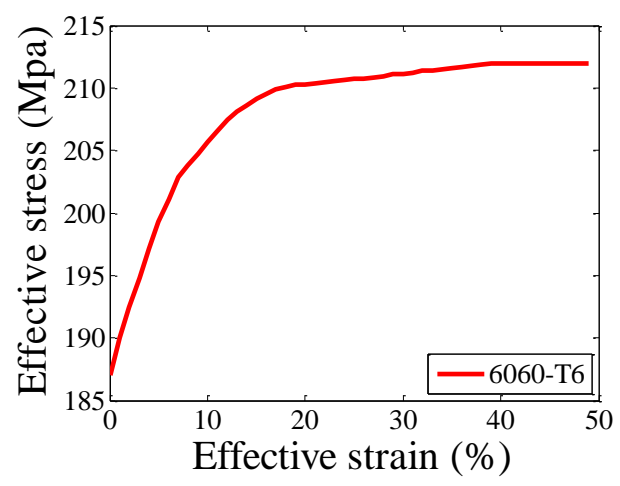

(c)

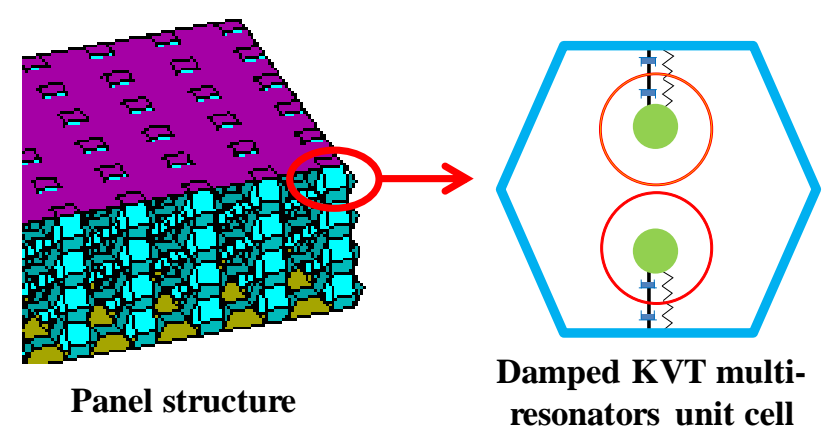

(d)

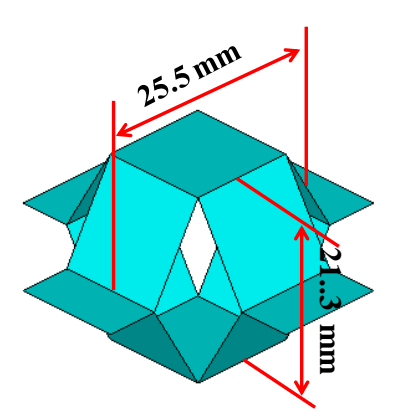

(e)

Fig.13. (a) The crash model of the three-dimensional sandwich panel structure. (b) The details of the three-dimensional sandwich panel structure. (c) The effective stress and effective strain curve of aluminum alloy 6060-T6. (d) The damped KVT multi-resonators unit cell. (e) The size of one decahedral honeycomb structure. 
Table 2. The damping coefficients of these dissipative microstructures

\begin{tabular}{ccccc}
\hline & $c_{2}(\mathrm{~N} / \mathrm{mm})$ & $c_{3}(\mathrm{~N} / \mathrm{mm})$ & $c_{4}(\mathrm{~N} / \mathrm{mm})$ & $c_{5}(\mathrm{~N} / \mathrm{mm})$ \\
\hline Group1 & 0.5 & 4.0 & 2.0 & 0.8 \\
Group2 & 5.0 & 25.0 & 15.0 & 7.0 \\
Group3 & 10.0 & 75.0 & 55.0 & 15.0 \\
Group4 & 30.0 & 180.0 & 140.0 & 37.0 \\
\hline
\end{tabular}

$c_{1}=0.5 \mathrm{Nm} / \mathrm{s}, c_{2}=4 \mathrm{Nm} / \mathrm{s}, c_{3}=2 \mathrm{Nm} / \mathrm{s}, c_{4}=0.8 \mathrm{Nm} / \mathrm{s}$
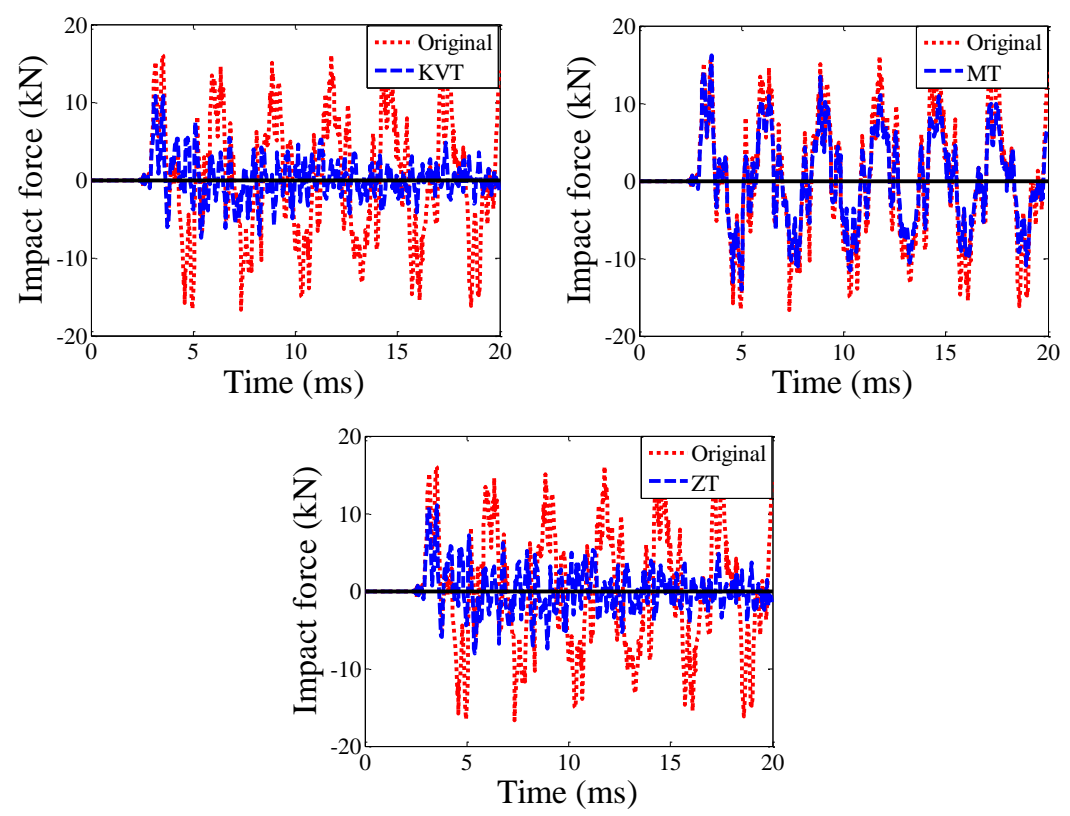

(a)
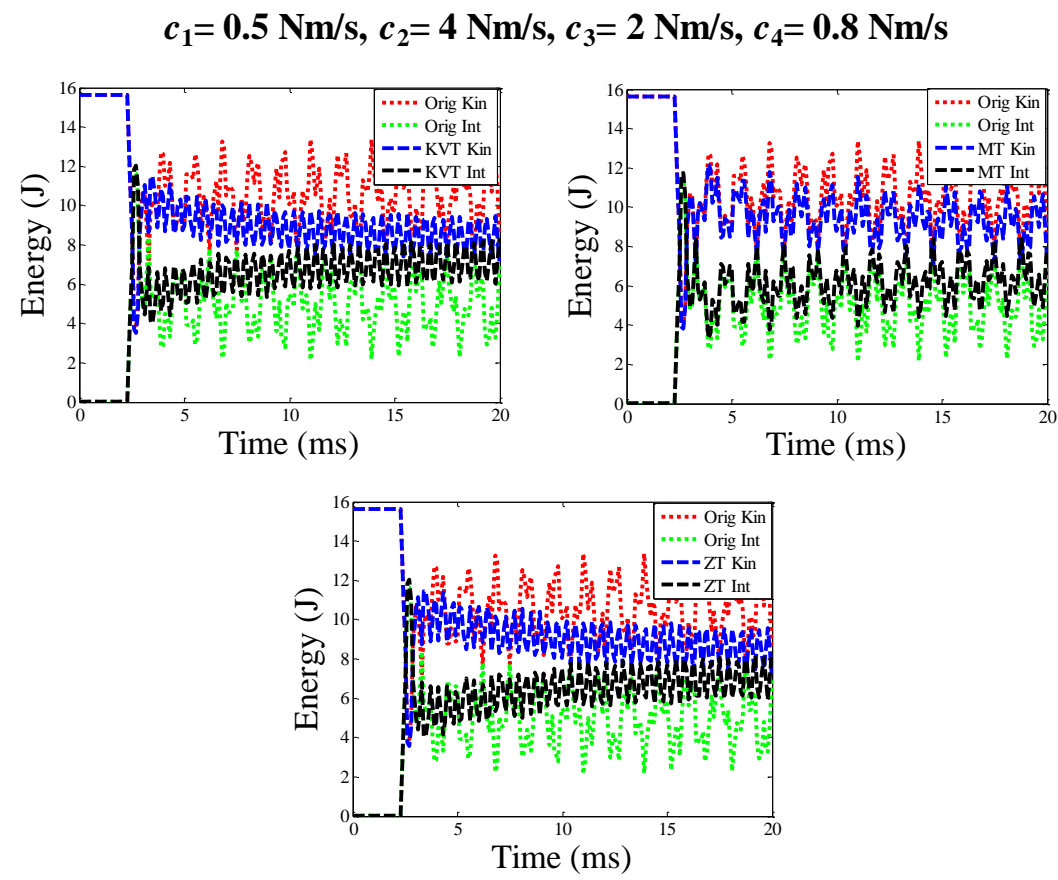

(b) 


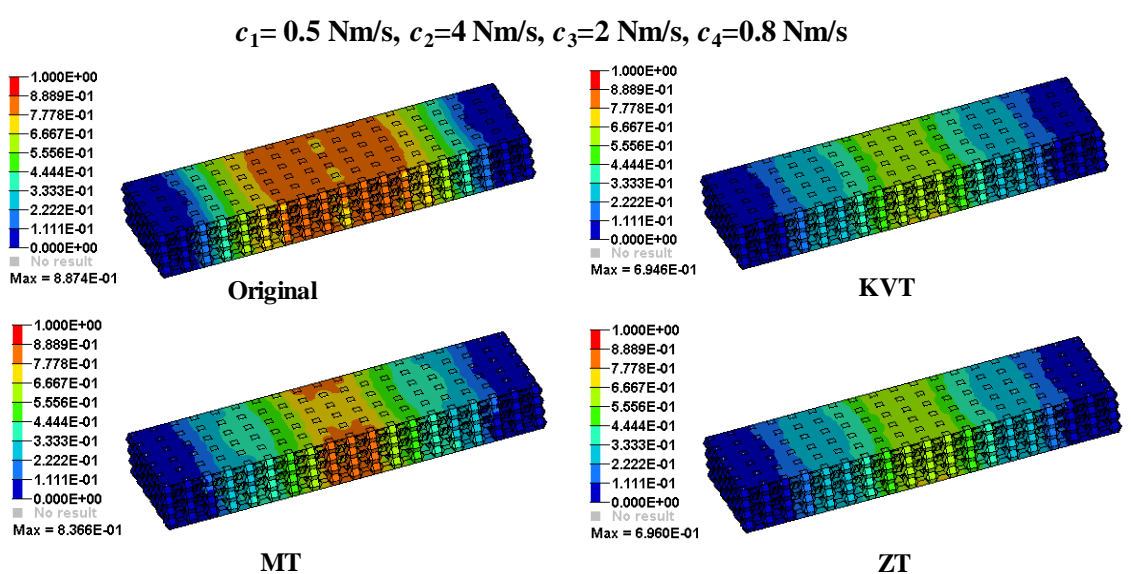

(c)

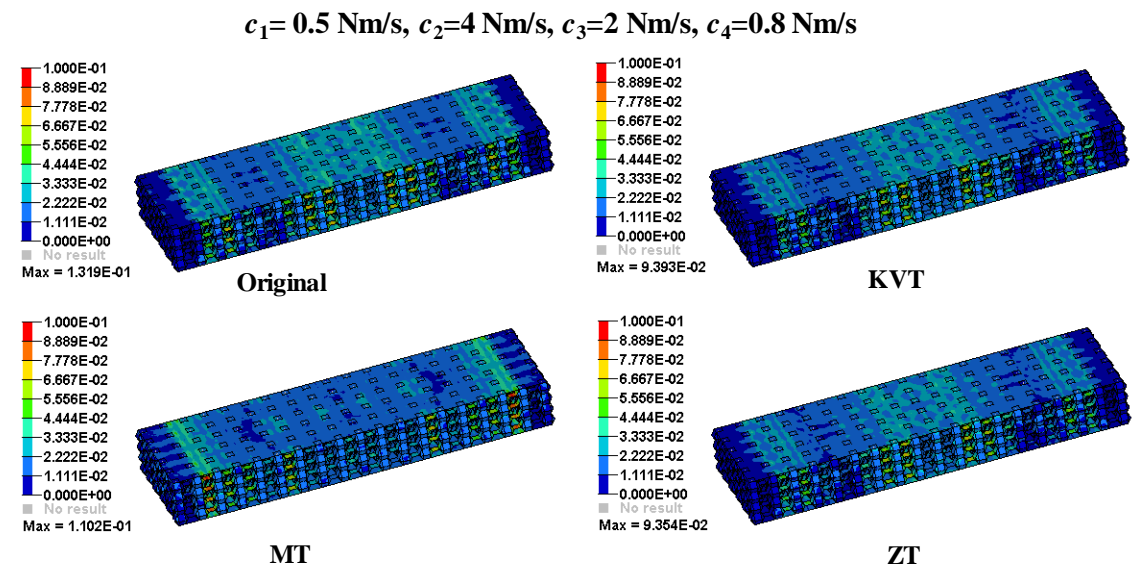

(d)

Fig.14. (a) The dynamic load responses with damping coefficients $c_{2}=0.5 \mathrm{~N} / \mathrm{mm}, c_{3}=$ $4 \mathrm{~N} / \mathrm{mm}, c_{4}=2 \mathrm{~N} / \mathrm{mm}$ and $c_{5}=0.8 \mathrm{~N} / \mathrm{mm}$. (b) The kinetic (Kin) energy and internal (Int) energy curves of the impact model with $c_{2}=0.5 \mathrm{~N} / \mathrm{mm}, c_{3}=4 \mathrm{~N} / \mathrm{mm}, c_{4}=2$ $\mathrm{N} / \mathrm{mm}$ and $c_{5}=0.8 \mathrm{~N} / \mathrm{mm}$. (c) The structural deformation contours of the sandwich panel structure with $c_{2}=0.5 \mathrm{~N} / \mathrm{mm}, c_{3}=4 \mathrm{~N} / \mathrm{mm}, c_{4}=2 \mathrm{~N} / \mathrm{mm}$ and $c_{5}=0.8 \mathrm{~N} / \mathrm{mm}$. (d) The structural stress nephograms of the panel structure with $c_{2}=0.5 \mathrm{~N} / \mathrm{mm}, c_{3}=4$ $\mathrm{N} / \mathrm{mm}, c_{4}=2 \mathrm{~N} / \mathrm{mm}$ and $c_{5}=0.8 \mathrm{~N} / \mathrm{mm}$.

Table. 3. Dynamic responses of these DAMs

\begin{tabular}{|c|c|c|c|c|}
\hline & & Initial peak force $(\mathrm{kN})$ & Maximum deformation (mm) & Maximum stress (Mpa) \\
\hline Original & & 16.00 & 0.89 & 131.90 \\
\hline \multirow{4}{*}{ KVT } & Group 1 & 10.87 & 0.69 & 93.93 \\
\hline & Group 2 & 9.45 & 0.63 & 88.83 \\
\hline & Group 3 & 8.57 & 0.58 & 85.04 \\
\hline & Group 4 & 7.63 & 0.52 & 77.39 \\
\hline \multirow{4}{*}{ MT } & Group 1 & 16.2 & 0.84 & 110.20 \\
\hline & Group 2 & 14.10 & 0.77 & 97.17 \\
\hline & Group 3 & 12.83 & 0.74 & 94.93 \\
\hline & Group 4 & 11.82 & 0.72 & 92.66 \\
\hline $\mathrm{ZT}$ & Group 1 & 11.09 & 0.70 & 93.87 \\
\hline
\end{tabular}




$\begin{array}{llll}\text { Group 2 } & 11.04 & 0.66 & 93.54 \\ \text { Group 3 } & 10.99 & 0.64 & 93.29 \\ \text { Group 4 } & 10.49 & 0.62 & 91.89\end{array}$

Fig.14 shows the plots of dynamic load curves, energy (internal energy and kinetic energy) curves, deformation contours and stress nephograms of the dissipative panel structure with damping coefficients $c_{2}=0.5 \mathrm{~N} / \mathrm{mm}, c_{3}=4 \mathrm{~N} / \mathrm{mm}, c_{4}=2 \mathrm{~N} / \mathrm{mm}$ and $c_{5}=0.8 \mathrm{~N} / \mathrm{mm}$. Table 3 shows the numerical results of the impact responses. As shown in Table 3 and Fig. 14, the initial peak load, maximum deformation and maximum stress of the original model are $16.0 \mathrm{kN}, 0.89 \mathrm{~mm}$ and $131.90 \mathrm{Mpa}$. From Figs. 14 (c) and (d), the structural deformation is mainly concentrated in the middle of the panel structure, and the stress is mainly distributed in the middle and the both sides of the structure. Correspondingly, in group 1, the peak loads of KVT, MT and ZT models are $10.87 \mathrm{kN}, 16.20 \mathrm{kN}$ and $11.09 \mathrm{kN}$, the maximum deformations of these models are $0.69 \mathrm{~mm}, 0.84 \mathrm{~mm}$, and $0.70 \mathrm{~mm}$, and their maximum structural stresses are 93.93 Mpa, 110.20 Mpa and 93.87 Mpa, respectively. Combined with Figs. 14 (a), (c) and (d), it is found that the peak load, structural deformation and structural stress are significantly alleviated for KVT and ZT models, and they are slightly reduced for MT model. Therefore, the attenuation effects of these impact responses are clearly realized through the use of these damped oscillators. Meanwhile, with the very small damping coefficients, the attenuation effects of KVT and ZT models are better than those of MT model, and ZT and KVT models have the similar impact results. In addition, the decline of structural deformation and structural stress means that structural damage is reduced, and these damped oscillators can also be applied to protect the structure.

Table. 4. Dynamic responses of the impact energy during $18 \mathrm{~ms}-20 \mathrm{~ms}$.

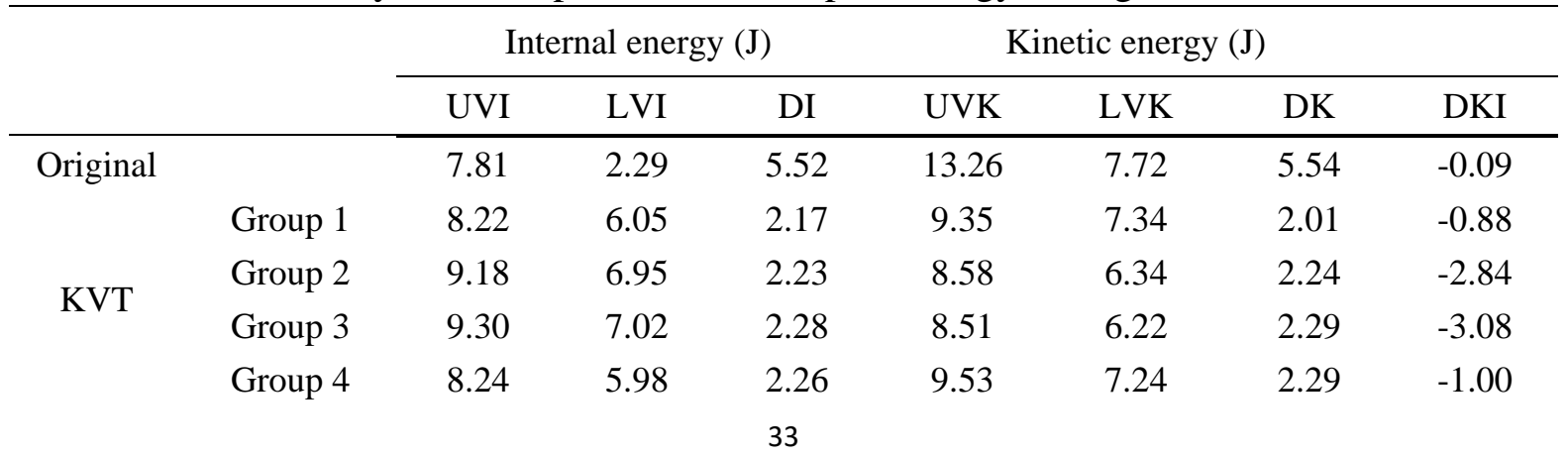




\begin{tabular}{lcccccccc} 
& Group 1 & 8.38 & 4.45 & 3.93 & 11.10 & 7.15 & 3.95 & -1.23 \\
MT & Group 2 & 8.35 & 5.53 & 2.82 & 10.00 & 7.17 & 2.83 & -1.18 \\
& Group 3 & 8.48 & 6.35 & 2.13 & 9.12 & 7.02 & 2.1 & -1.46 \\
& Group 4 & 8.25 & 6.08 & 2.17 & 9.40 & 7.26 & 2.14 & -0.99 \\
& Group 1 & 8.08 & 6.02 & 2.06 & 9.49 & 7.45 & 2.04 & -0.63 \\
\multirow{2}{*}{ ZT } & Group 2 & 8.13 & 6.1 & 2.03 & 9.56 & 7.38 & 2.18 & -0.75 \\
& Group 3 & 7.91 & 5.39 & 2.52 & 10.11 & 7.59 & 2.52 & -0.32 \\
& Group 4 & 7.82 & 5.13 & 2.69 & 10.36 & 7.68 & 2.68 & -0.14 \\
\hline
\end{tabular}

Table 4 shows the numerical results of kinetic energy and internal energy during $18 \mathrm{~ms}$ - $20 \mathrm{~ms}$. The upper value of internal energy (UVI) and the lower value of internal energy (LVI) mean that the maximum and minimum values of the internal energy curve during $18 \mathrm{~ms}$ - $20 \mathrm{~ms}$, respectively. Analogously, the upper value of kinetic energy (UVK) and the lower value of kinetic energy (LVK) stand for the maximum and minimum values of the kinetic energy curve during $18 \mathrm{~ms}-20 \mathrm{~ms}$. The difference between UVI and LVI is the distance between the upper and lower values of the internal energy curve (DI), and the value of UVK minus LVK is the distance between the upper and lower values of the kinetic energy curve (DK). The distance between the minimum value of kinetic energy curve and the maximum value of internal energy curve (DKI) during $18 \mathrm{~ms}-20 \mathrm{~ms}$ is the difference between LVK and UVI. It should be noted that the values of DI and DK are always positive, and a negative value of DKI means that there is an overlap between the internal energy and the kinetic energy curves. The decrease of DI, DK and DKI implies the improvement of the dissipation of impact energy.

From Figs. 14 (a), (b) and Table 4, the dynamic load, kinetic energy and internal energy curves of original model keep oscillating with time. The initial kinetic energy and initial internal energy are $15.62 \mathrm{~J}$ and $0 \mathrm{~J}$, and the values of DI, DK and DKI are $5.52 \mathrm{~J}, 5.54 \mathrm{~J}$ and $-0.09 \mathrm{~J}$, respectively. Obviously, the values of DI, DK and DKI of the original model are large, and the impact energy is not sufficiently absorbed. For the KVT, MT and ZT models, their amplitudes of dynamic load and kinetic energy drop with time, and their internal energy amplitude rises with time. This means these damped oscillators can dissipate the impact energy very well. Furthermore, the drop 
rates of the dynamic load and kinetic energy curves as well as the rise rate of the internal energy curve of KVT and ZT models are faster than those of MT model. The values of DI, DK and DKI of KVT model in group 1 are $2.17 \mathrm{~J}, 2.01 \mathrm{~J}$ and $-0.88 \mathrm{~J}$, these values of MT model are $3.93 \mathrm{~J}, 3.95 \mathrm{~J}$ and $-1.23 \mathrm{~J}$, and their values in ZT model are $2.06 \mathrm{~J}, 2.04 \mathrm{~J}$ and $-0.63 \mathrm{~J}$, respectively. KVT and $\mathrm{ZT}$ models have the similar values of DI, DK and DKI, and their values of DI and DK are smaller than those of MT. Therefore, with current damping coefficients, the dissipation effect of KVT is similar as ZT and significantly better than that of MT. The impact energy can be quickly absorbed by KVT and ZT oscillators. These numerical results clear prove that the DAMs can significantly attenuate the crash stress wave and dissipate the impact energy based on their properties of negative effective mass and effective metadamping. In addition, from Fig.14 and Tables 3, 4, the attenuation and dissipation effects of MT oscillators are poor with the very small damping coefficients. This is because that small damping reduces the connection stiffness between the oscillators, and the motion intensity of oscillator is also alleviated. Therefore, the great attenuation and dissipation effects of MT oscillators require a relatively large damping coefficient.

\subsection{Parameter analyses of the attenuation and dissipation effects of the dissipative three-dimensional sandwich panel model}

The damping coefficients in groups 2 - 4 are applied in the dissipative panel structure, respectively. Figs. 15 - 18 and Tables 3, 4 show the plot and numerical results of the impact response for each group of damping parameters. As shown in Table 3 and Figs. 15 - 18, the peak dynamic loads of KVT, MT and ZT models with damping coefficients $c_{2}=5 \mathrm{~N} / \mathrm{mm}, c_{3}=25 \mathrm{~N} / \mathrm{mm}, c_{4}=15 \mathrm{~N} / \mathrm{mm}$ and $c_{5}=7 \mathrm{~N} / \mathrm{mm}$ are $9.45 \mathrm{kN}, 14.10 \mathrm{kN}$ and $11.04 \mathrm{kN}$, their maximum deformations are $0.63 \mathrm{~mm}, 0.70 \mathrm{~mm}$ and $0.66 \mathrm{~mm}$, and their maximum stresses are 88.83 Mpa, 97.17 Mpa and 93.54 Mpa. In group 3, the maximum loads, deformations and stresses of KVT, MT and ZT models are $8.57 \mathrm{kN}, 12.83 \mathrm{kN}, 10.99 \mathrm{kN}, 0.58 \mathrm{~mm}, 0.74 \mathrm{~mm}, 0.64 \mathrm{~mm}, 85.04 \mathrm{Mpa}$, 
94.93 Mpa and 93.29 Mpa. In group 4, their peak collision responses are $7.63 \mathrm{kN}$, $11.82 \mathrm{kN}, 10.49 \mathrm{kN}, 0.52 \mathrm{~mm}, 0.72 \mathrm{~mm}$ and $0.62 \mathrm{~mm}, 77.39 \mathrm{Mpa}, 92.66 \mathrm{Mpa}$ and 91.89 Mpa, respectively. The values of these impact results of KVT, MT and ZT models reduce as the damping increase, which means the attenuation effects of these metamaterials are improved. The best attenuation effects of these three kinds of DAMs are achieved with the damping coefficients $c_{2}=30 \mathrm{~N} / \mathrm{mm}, c_{3}=180 \mathrm{~N} / \mathrm{mm}, c_{4}=$ $140 \mathrm{~N} / \mathrm{mm}$ and $c_{5}=37 \mathrm{~N} / \mathrm{mm}$. The maximum attenuation rates of peak load, maximum deformation and maximum stress of KVT model are $52.31 \%, 41.57 \%$ and 41.32\%. For MT model, its maximum attenuation rates are $26.13 \%, 19.10 \%$ and $29.75 \%$, and the peak mitigation rates of ZT model are $34.44 \%, 30.34 \%$ and $30.33 \%$. With the high damping coefficients, the attenuation effects of KVT are better than those of MT and ZT, and the performances of MT is the worst among these damped models. In addition, with the increase of the damping coefficients, the impact responses of KVT and MT models decline faster than those of ZT oscillators.

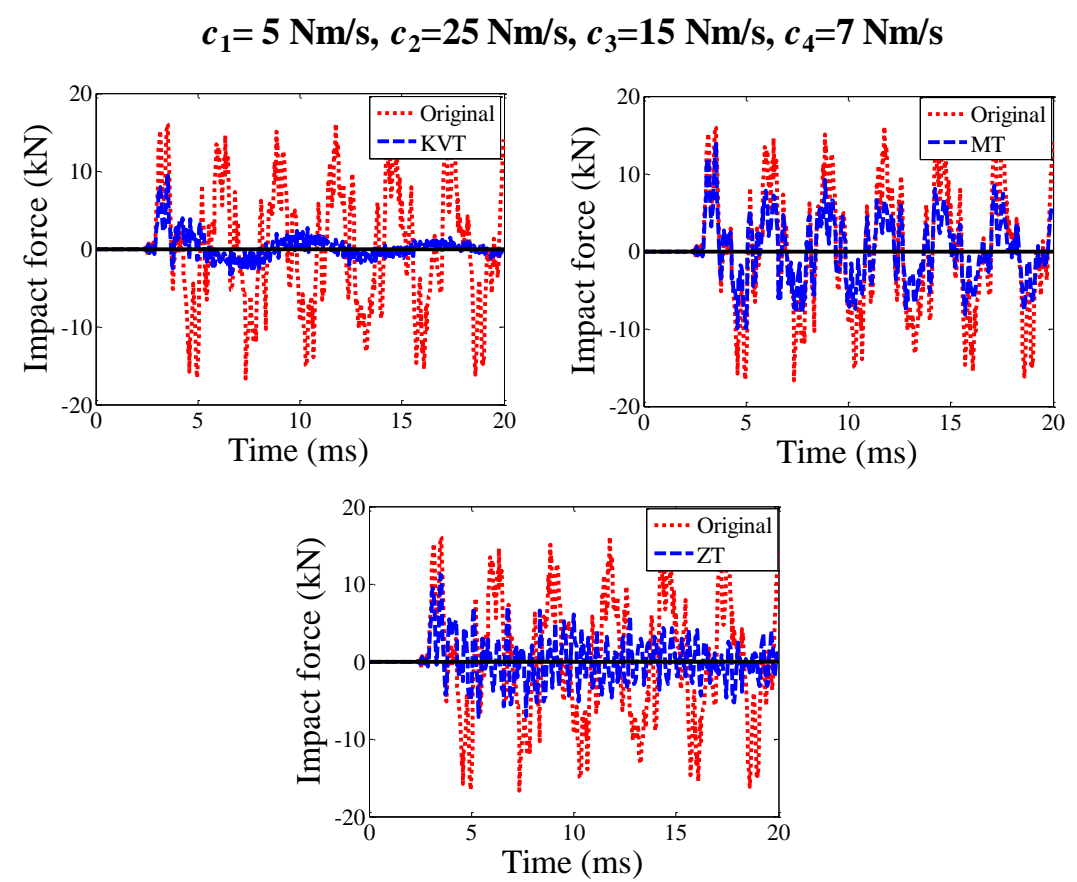

(a) 
$c_{1}=5 \mathrm{Nm} / \mathrm{s}, c_{2}=25 \mathrm{Nm} / \mathrm{s}, c_{3}=15 \mathrm{Nm} / \mathrm{s}, c_{4}=7 \mathrm{Nm} / \mathrm{s}$
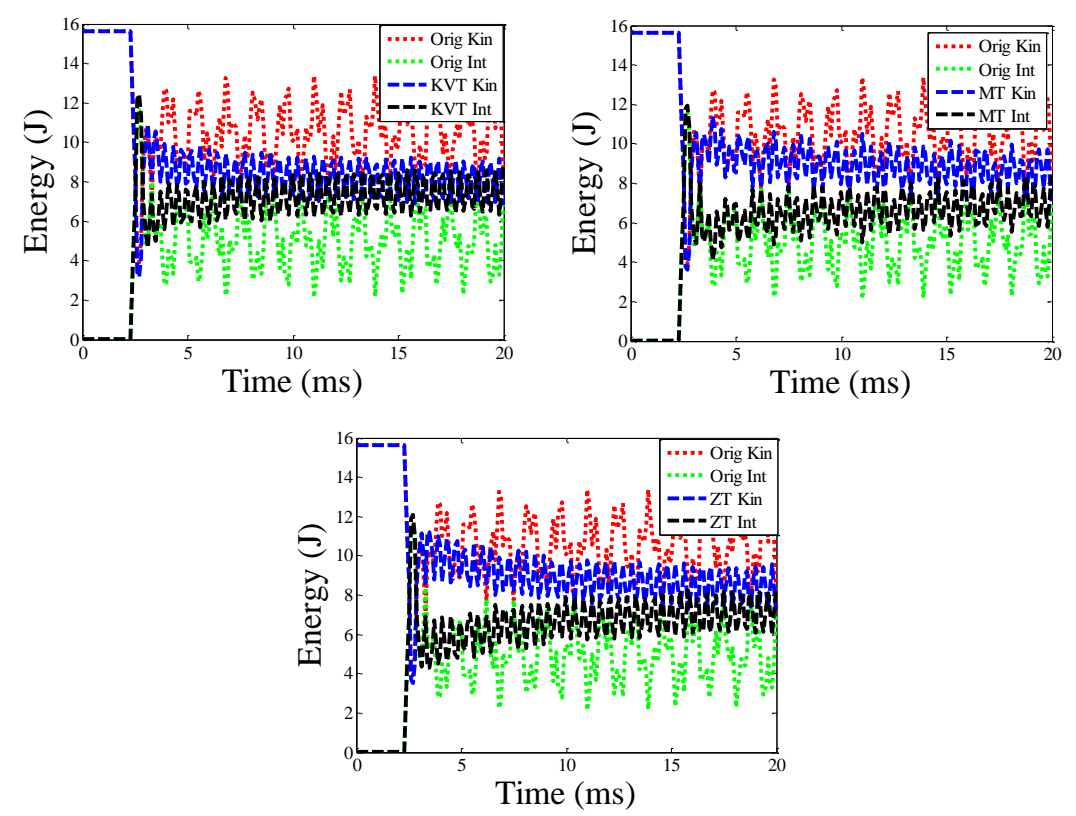

(c)

$c_{1}=5 \mathrm{Nm} / \mathrm{s}, c_{2}=25 \mathrm{Nm} / \mathrm{s}, c_{3}=15 \mathrm{Nm} / \mathrm{s}, c_{4}=7 \mathrm{Nm} / \mathrm{s}$
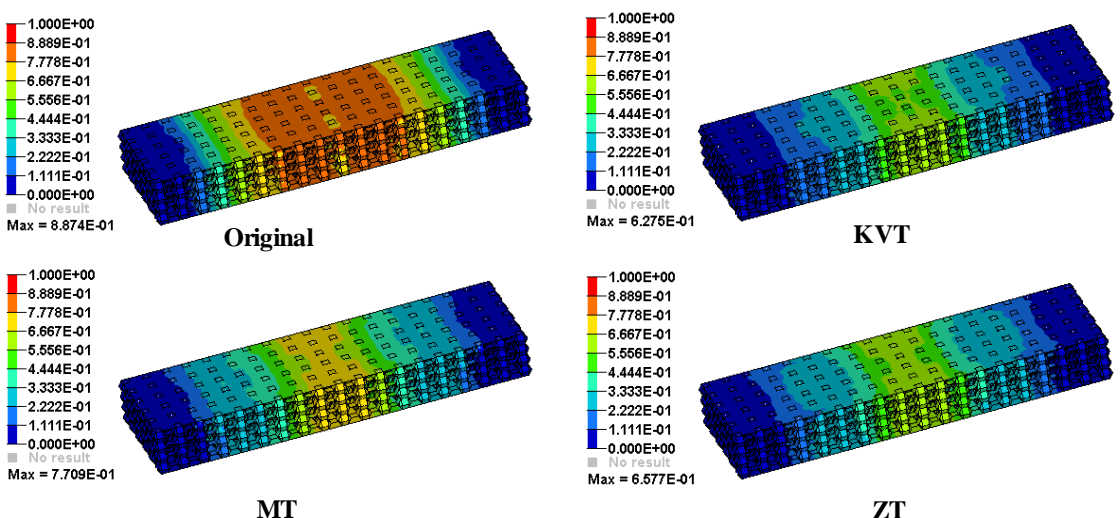

(c)

$c_{1}=5 \mathrm{Nm} / \mathrm{s}, c_{2}=25 \mathrm{Nm} / \mathrm{s}, c_{3}=15 \mathrm{Nm} / \mathrm{s}, c_{4}=7 \mathrm{Nm} / \mathrm{s}$
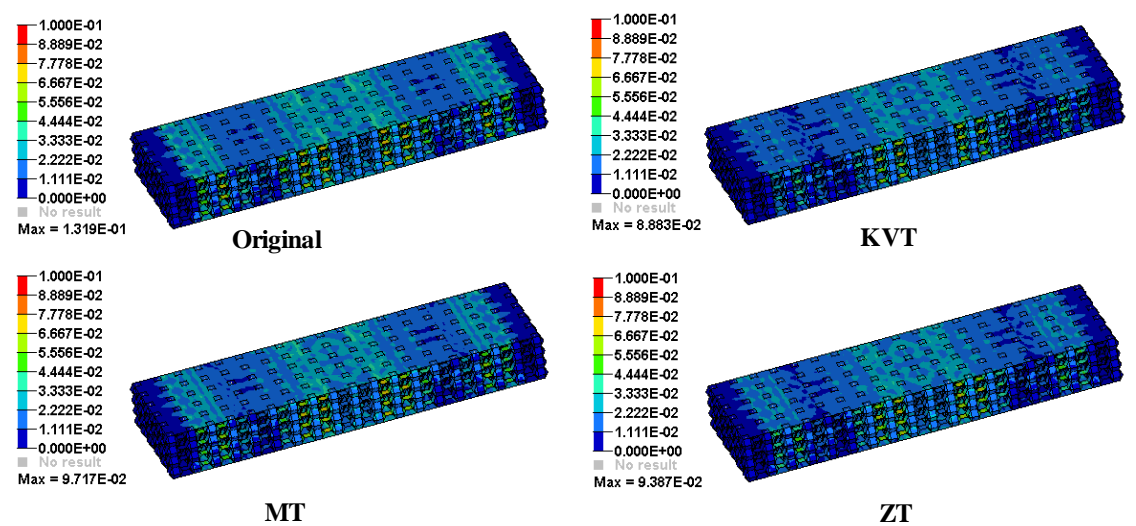

(d)

Fig.15. (a) The dynamic load responses with damping coefficients $c_{2}=5 \mathrm{~N} / \mathrm{mm}, c_{3}=$ $25 \mathrm{~N} / \mathrm{mm}, c_{4}=15 \mathrm{~N} / \mathrm{mm}$ and $c_{5}=7 \mathrm{~N} / \mathrm{mm}$. (b) The kinetic energy and internal energy 
curves of the impact model with $c_{2}=5 \mathrm{~N} / \mathrm{mm}, c_{3}=25 \mathrm{~N} / \mathrm{mm}, c_{4}=15 \mathrm{~N} / \mathrm{mm}$ and $c_{5}=$ $7 \mathrm{~N} / \mathrm{mm}$. (c) The structural deformation contours of the sandwich panel structure with $c_{2}=5 \mathrm{~N} / \mathrm{mm}, c_{3}=25 \mathrm{~N} / \mathrm{mm}, c_{4}=15 \mathrm{~N} / \mathrm{mm}$ and $c_{5}=7 \mathrm{~N} / \mathrm{mm}$. (d) The structural stress nephograms of the panel structure with $c_{2}=5 \mathrm{~N} / \mathrm{mm}, c_{3}=25 \mathrm{~N} / \mathrm{mm}, c_{4}=15 \mathrm{~N} / \mathrm{mm}$ and $c_{5}=7 \mathrm{~N} / \mathrm{mm}$.
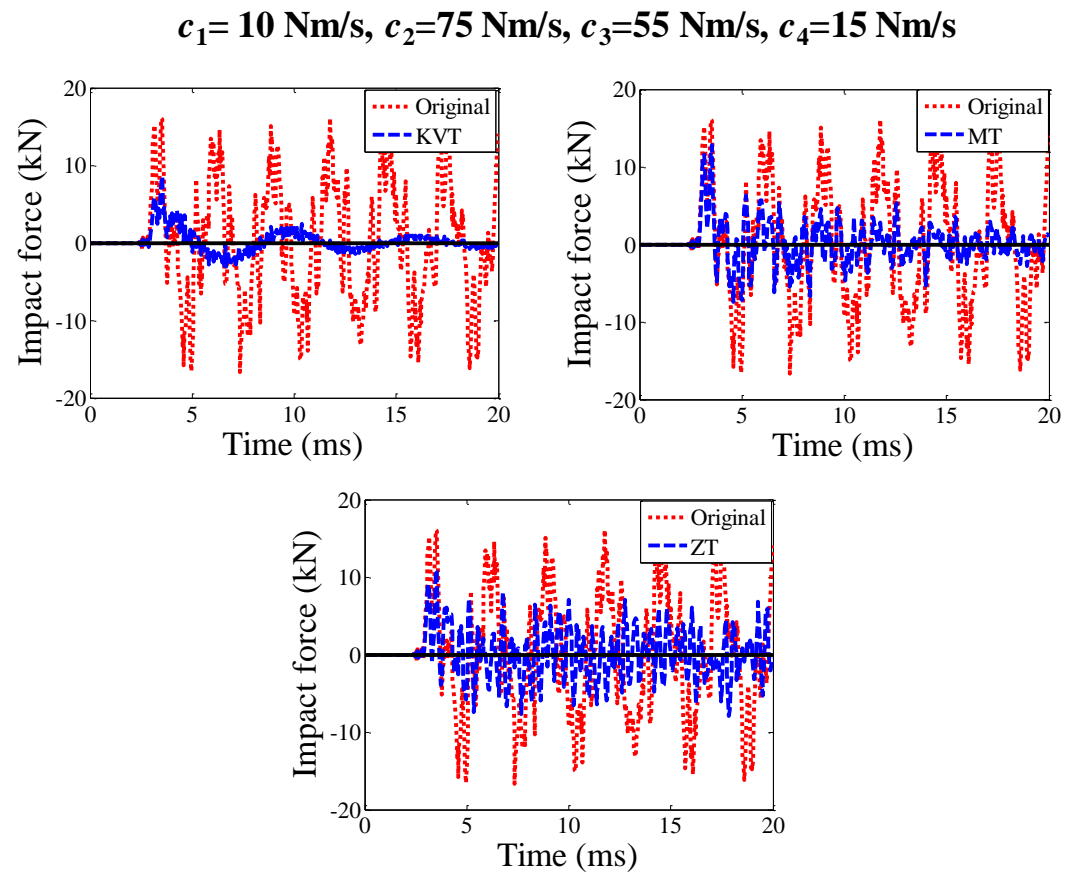

(a)

$c_{1}=10 \mathrm{Nm} / \mathrm{s}, c_{2}=75 \mathrm{Nm} / \mathrm{s}, c_{3}=55 \mathrm{Nm} / \mathrm{s}, c_{4}=15 \mathrm{Nm} / \mathrm{s}$
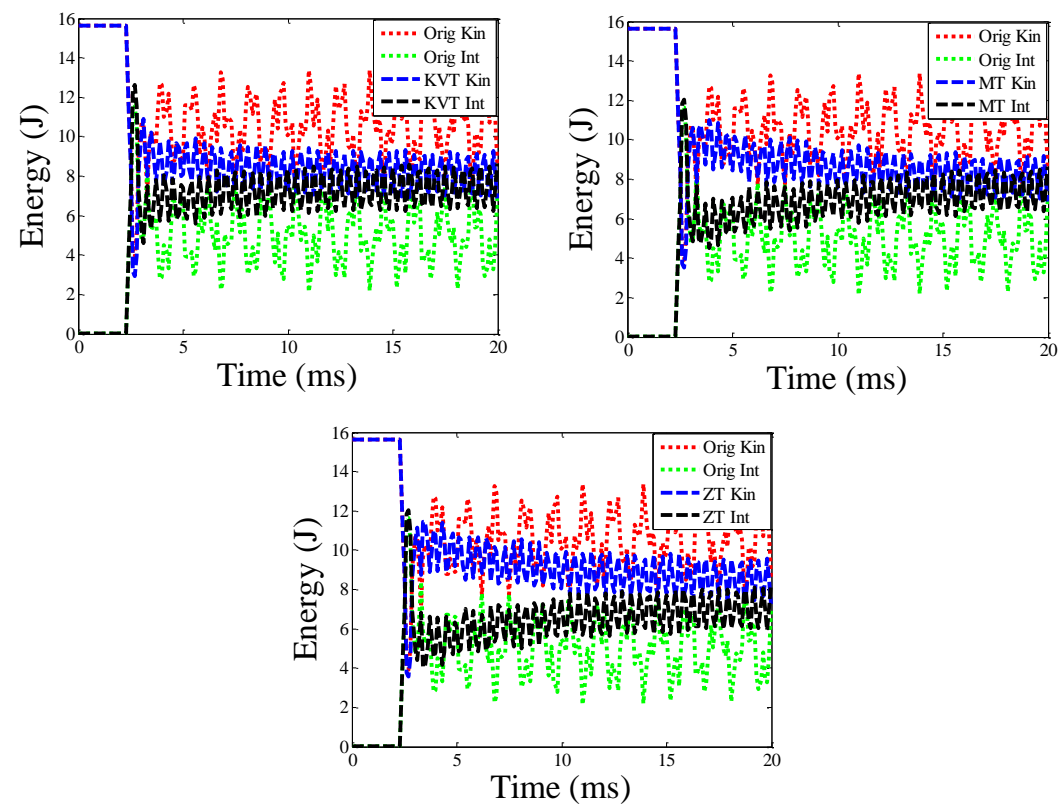

(b) 
$c_{1}=10 \mathrm{Nm} / \mathrm{s}, c_{2}=75 \mathrm{Nm} / \mathrm{s}, c_{3}=55 \mathrm{Nm} / \mathrm{s}, c_{4}=15 \mathrm{Nm} / \mathrm{s}$

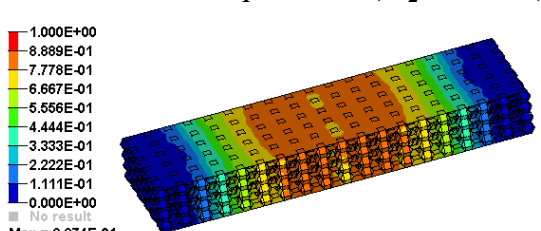

Original

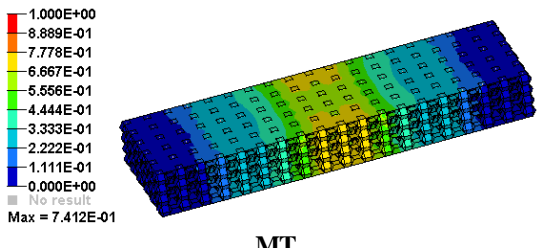

MT

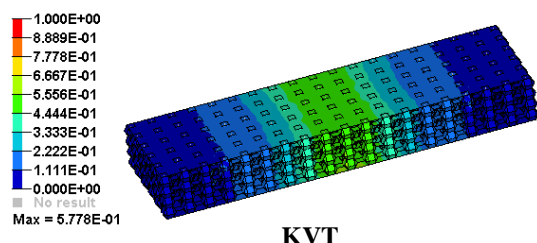

KVT

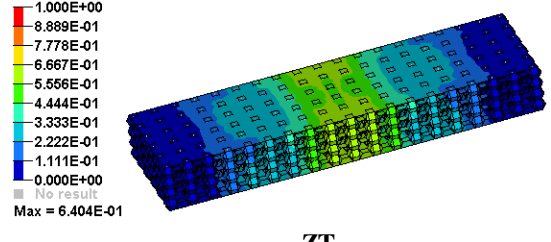

ZT

(c)

$c_{1}=10 \mathrm{Nm} / \mathrm{s}, c_{2}=75 \mathrm{Nm} / \mathrm{s}, c_{3}=55 \mathrm{Nm} / \mathrm{s}, c_{4}=15 \mathrm{Nm} / \mathrm{s}$
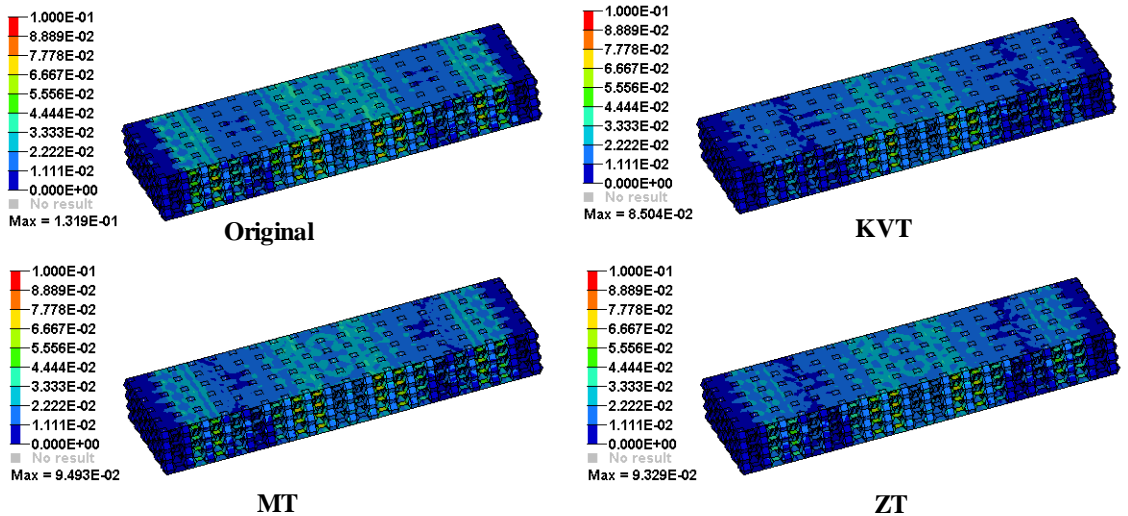

(d)

Fig.16. (a) The dynamic load responses with damping coefficients $c_{2}=10 \mathrm{~N} / \mathrm{mm}, c_{3}=$ $75 \mathrm{~N} / \mathrm{mm}, c_{4}=55 \mathrm{~N} / \mathrm{mm}$ and $c_{5}=15 \mathrm{~N} / \mathrm{mm}$. (b) The kinetic energy and internal energy curves of the impact model with $c_{2}=10 \mathrm{~N} / \mathrm{mm}, c_{3}=75 \mathrm{~N} / \mathrm{mm}, c_{4}=55 \mathrm{~N} / \mathrm{mm}$ and $c_{5}=15 \mathrm{~N} / \mathrm{mm}$. (c) The structural deformation contours of the sandwich panel structure with $c_{2}=10 \mathrm{~N} / \mathrm{mm}, c_{3}=75 \mathrm{~N} / \mathrm{mm}, c_{4}=55 \mathrm{~N} / \mathrm{mm}$ and $c_{5}=15 \mathrm{~N} / \mathrm{mm}$. (d) The structural stress nephograms of the panel structure with $c_{2}=10 \mathrm{~N} / \mathrm{mm}, c_{3}=75 \mathrm{~N} / \mathrm{mm}$, $c_{4}=55 \mathrm{~N} / \mathrm{mm}$ and $c_{5}=15 \mathrm{~N} / \mathrm{mm}$. 

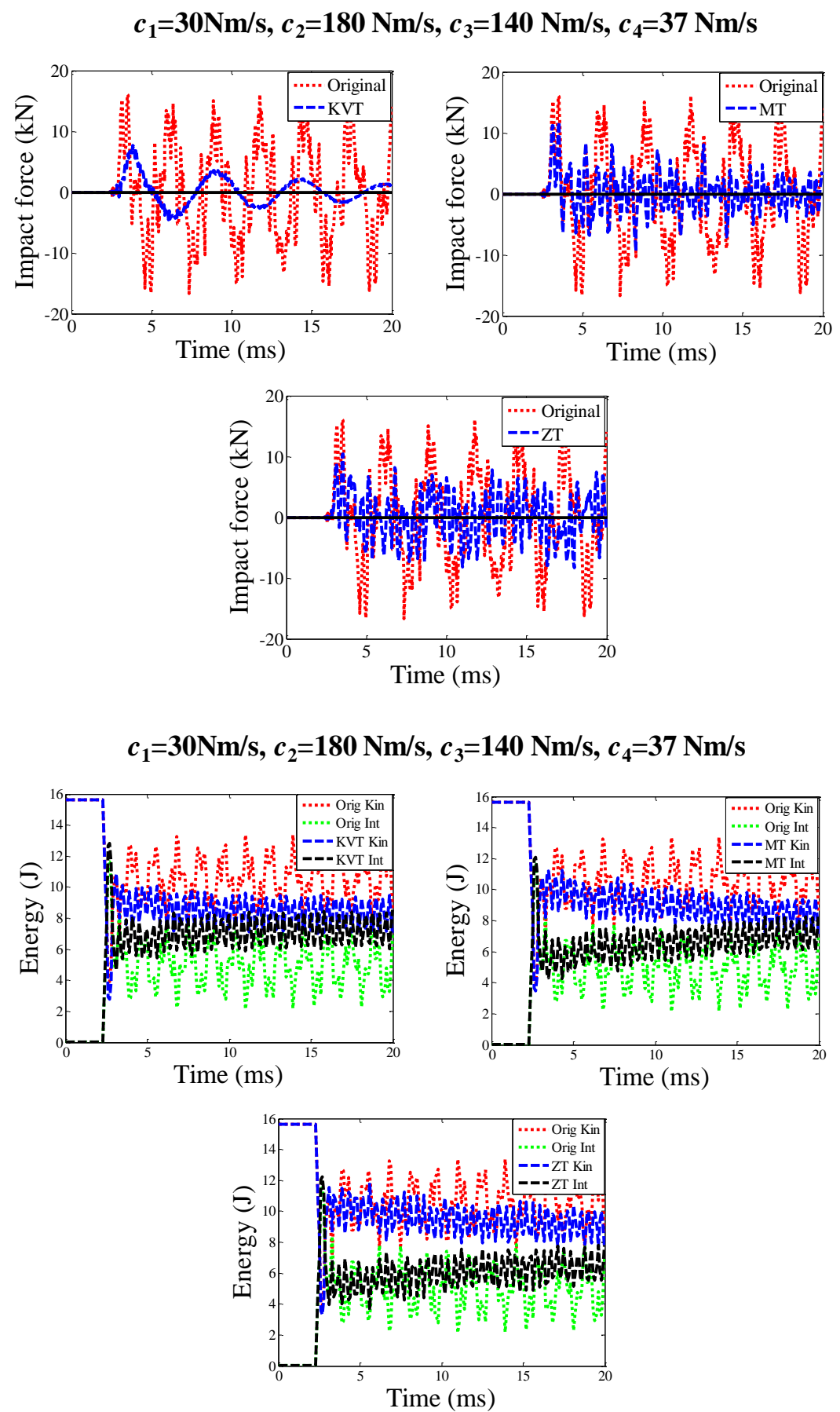

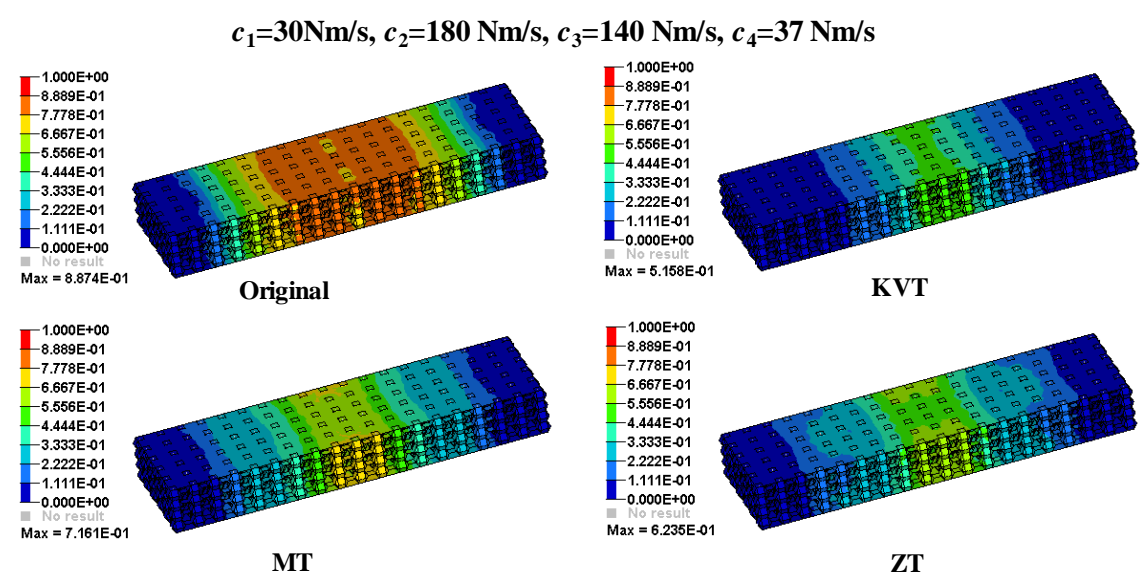

$c_{1}=30 \mathrm{Nm} / \mathrm{s}, c_{2}=180 \mathrm{Nm} / \mathrm{s}, c_{3}=140 \mathrm{Nm} / \mathrm{s}, c_{4}=37 \mathrm{Nm} / \mathrm{s}$
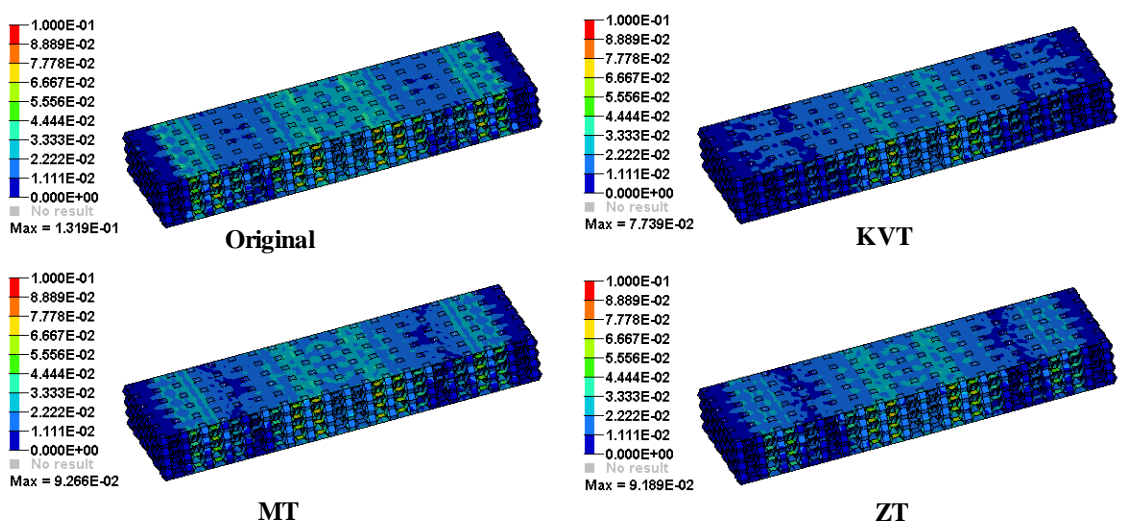

Fig.17. (a) The dynamic load responses with damping coefficients $c_{2}=30 \mathrm{~N} / \mathrm{mm}, c_{3}=$ $180 \mathrm{~N} / \mathrm{mm}, c_{4}=140 \mathrm{~N} / \mathrm{mm}$ and $c_{5}=37 \mathrm{~N} / \mathrm{mm}$. (b) The kinetic energy and internal energy curves of the impact model with $c_{2}=30 \mathrm{~N} / \mathrm{mm}, c_{3}=180 \mathrm{~N} / \mathrm{mm}, c_{4}=140$ $\mathrm{N} / \mathrm{mm}$ and $c_{5}=37 \mathrm{~N} / \mathrm{mm}$. (c) The structural deformation contours of the sandwich panel structure with $c_{2}=30 \mathrm{~N} / \mathrm{mm}, c_{3}=180 \mathrm{~N} / \mathrm{mm}, c_{4}=140 \mathrm{~N} / \mathrm{mm}$ and $c_{5}=37$ $\mathrm{N} / \mathrm{mm}$. (d) The structural stress nephograms of the panel structure with $c_{2}=30 \mathrm{~N} / \mathrm{mm}$, $c_{3}=180 \mathrm{~N} / \mathrm{mm}, c_{4}=140 \mathrm{~N} / \mathrm{mm}$ and $c_{5}=37 \mathrm{~N} / \mathrm{mm}$.
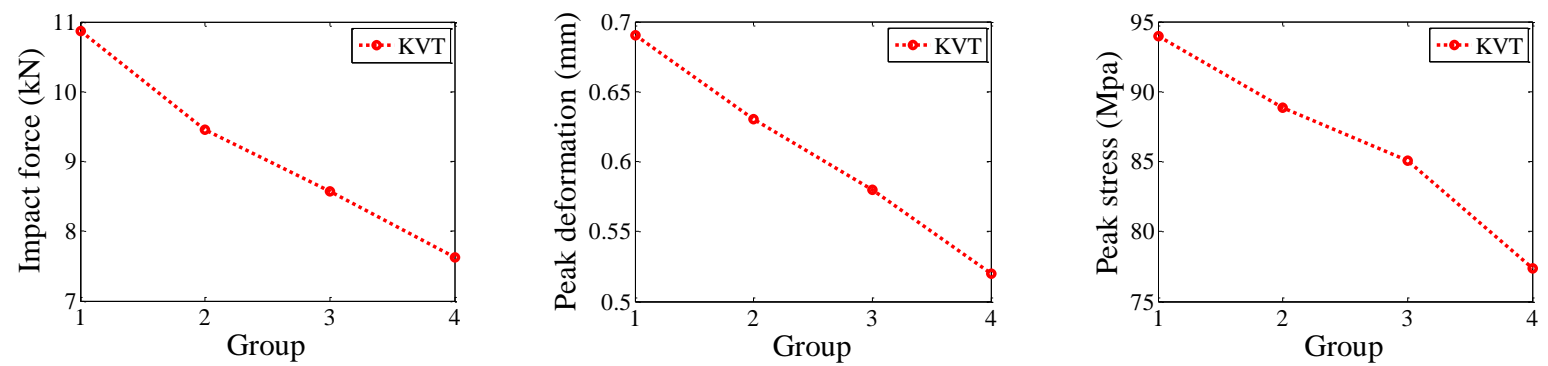

(a) 

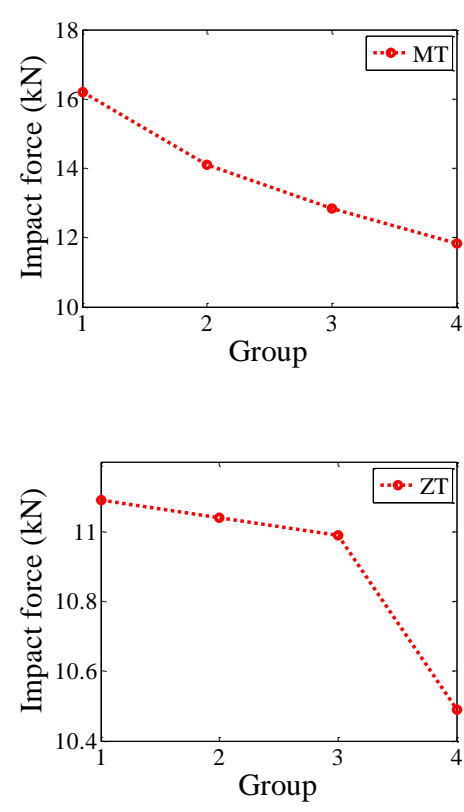

Fig. 18. (a) The plots of peak load, maximum structural deformation and maximum structural stress of KVT model with each group of damping coefficients. (b) The plots of these impact responses of MT model. (c) The plots of these collision results of ZT model.

As shown in Figs. (14) - (17), the rates of change of dynamic load, kinetic energy and internal energy increase first and then decrease with the increase of damping. From Table 4, the values of DI, LVI and DKI are $2.23 \mathrm{~J}, 2.24 \mathrm{~J}$ and $-2.84 \mathrm{~J}$ for KVT in group 2, $2.28 \mathrm{~J}, 2.29 \mathrm{~J}$ and $-3.08 \mathrm{~J}$ for $\mathrm{KVT}$ in group 3, $2.26 \mathrm{~J}, 2.29 \mathrm{~J}$ and $-1.00 \mathrm{~J}$ for KVT in group 4, $2.82 \mathrm{~J}, 2.83 \mathrm{~J}$ and $-1.18 \mathrm{~J}$ for MT in group 2, 2.13 J, 2.10 J and -1.46 $\mathrm{J}$ for MT in group 3, $2.17 \mathrm{~J}, 2.14 \mathrm{~J}$ and $-0.99 \mathrm{~J}$ for MT in group 4, $2.03 \mathrm{~J}, 2.18 \mathrm{~J}$ and $-0.75 \mathrm{~J}$ for ZT in group 2, 2.52 J, 2.52 J and $-0.32 \mathrm{~J}$ for ZT in group 3, and 2.69 J, 2.68 $\mathrm{J}$ and $-0.14 \mathrm{~J}$ for ZT in group 4. Fig. 19 shows the plots of DI, LVI and DKI for each group of damping parameters with KVT, MT and ZT oscillators. From Table 4 and Fig. 19, the trend of the values of DKI decreases first and then increases with the increase of damping coefficients, which means that the dissipation effects of the impact energy of KVT, MT and ZT models increase first and then decrease. The main reasons of these phenomena are summarized as follows. First, the increase of damping enhances the values of effective metadamping coefficient of MT microstructure while reducing its band gap regions. Second, with the increase of damping, the band gap width of effective metadamping of KVT and ZT microstructures are expended, and 
their amplitudes are declined. In addition, these analyses imply that more collision energy can be dissipated by the reasonable matching of damping parameters of these DAMs.
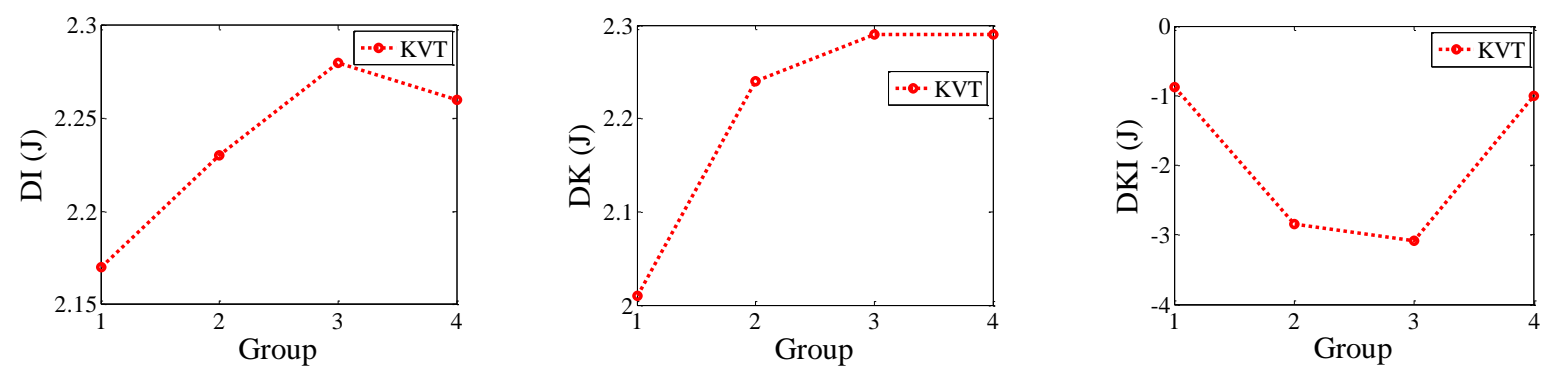

(a)
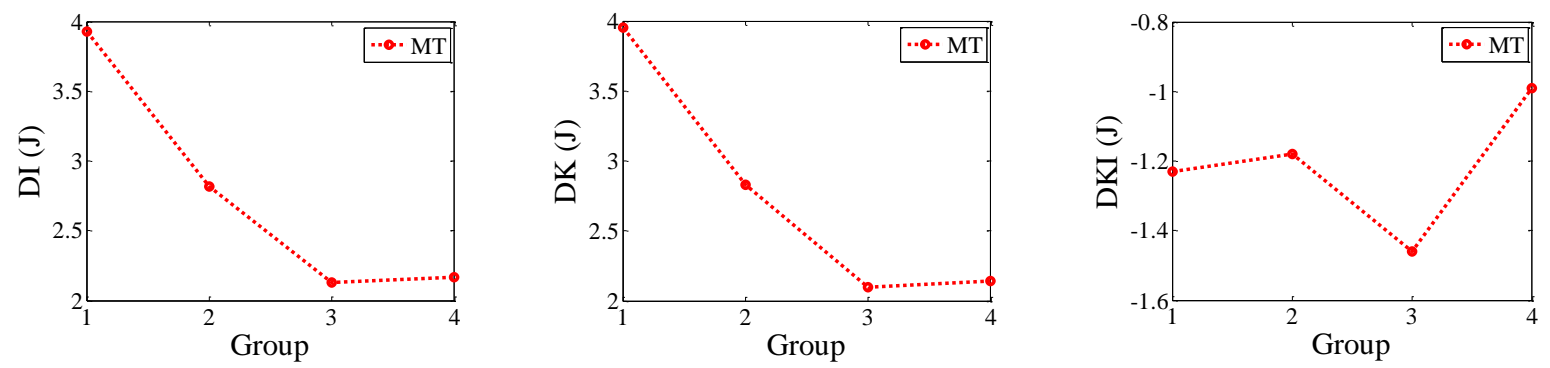

(b)
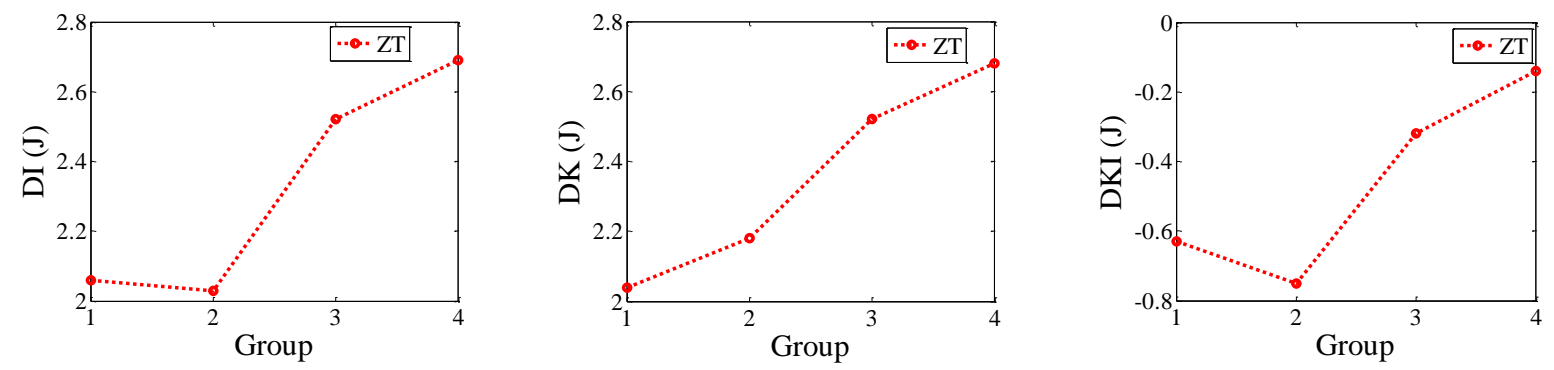

(c)

Fig. 19. (a) The plots of DI, DK and DKI of KVT model with each group of damping coefficients. (b) The plots of DI, DK and DKI of MT model. (c) The plots of DI, DK and DKI of ZT model.

Furthermore, the rates of change of DI and DK are small for KVT, while its DKI changes significantly with the increase of damping. For MT model, with the increase of damping, the values of DI and DK are changed obviously, while the rate of change of DKI is small. Regarding ZT model, with the increase of damping, all these three parameters have a small rate of change. Therefore, the dissipation effects of KVT and MT models are significantly changed with the increase of damping, while the influence of damping on the absorption of the impact energy is less for ZT model. In 
addition, from Table 3 and Figs. 14 - 18, the change of peak load of ZT model is also not obvious. Therefore, damping has less effect on the attenuation and dissipation of ZT oscillators. In addition, referring to the numerical and plot results of internal energy, kinetic energy and dynamic load, the maximum energy dissipation occurs for ZT and KVT models when the damping coefficients are $c_{2}=5 \mathrm{~N} / \mathrm{mm}, c_{3}=25 \mathrm{~N} / \mathrm{mm}$, $c_{4}=15 \mathrm{~N} / \mathrm{mm}$ and $c_{5}=7 \mathrm{~N} / \mathrm{mm}$. The dissipation effect of impact energy of MT oscillators can be significantly enhanced when the damping coefficients are set as $c_{2}=$ $10 \mathrm{~N} / \mathrm{mm}, c_{3}=75 \mathrm{~N} / \mathrm{mm}, c_{4}=55 \mathrm{~N} / \mathrm{mm}$ and $c_{5}=15 \mathrm{~N} / \mathrm{mm}$.

These results can be summarized as follows. (1) KVT oscillators have the best performance in mitigating and dissipating the impact energy. (2) With the very small damping coefficients, the attenuation and dissipation effects of KVT and ZT are similar with each other, and the performances of MT are poor. (3) Damping has less influence on the performances of ZT model. (4) The dissipation effects of KVT, MT and ZT increase first and then decrease with the increase of damping coefficients.

\section{Conclusion}

In this paper, the multi-resonator dissipative acoustic metamaterials (DAMs) with Kelvin-Voigt-type (KVT), Maxwell-type (MT) and Zener-type (ZT) oscillators are studied because of their remarkable characteristics for stress waves attenuation and energy dissipation. These DAMs are applied in the collision conditions to reduce the dynamic load, structural deformation and structural stress and to absorb the impact energy based on their properties of negative effective mass and effective metadamping. KVT oscillators show the best performances in load mitigation and energy absorption. Regarding MT microstructure, the proper selection of damping can both improve its attenuation and dissipation effects. The influence of damping on the performances of ZT oscillators is less than that of KVT and MT models.

\section{References}

1. Chen H, Li XP, Chen YY, Huang GL. Wave propagation and absorption of sandwich beams containing interior dissipative multi-resonators. Ultrasonics 2017,76:99-108. 
2. Li Y, Zhu L, Chen T. Plate-type elastic metamaterials for low-frequency broadband elastic wave attenuation. Ultrasonics 2017,73:34-42.

3. Zhou YH, Wei PJ, Tang QH. Continuum model of a one-dimensional lattice of metamaterials. Acta Mechanica 2016,227:2361-2376.

4. $\quad \mathrm{He} \mathrm{Z}, \mathrm{Hu}$ J, Li E. An uncertainty model of acoustic metamaterials with random parameters. Computational Mechanics 2018:1-14.

5. Li E, He Z, Wang G, Jong Y. Fundamental study of mechanism of band gap in fluid and solid/fluid phononic crystals. Advances in Engineering Software 2018,121:167-177.

6. Li E, He Z, Wang G, Liu G. An efficient algorithm to analyze wave propagation in fluid/solid and solid/fluid phononic crystals. Computer Methods in Applied Mechanics and Engineering 2018,333:421-442.

7. Xiao X, He ZC, Li E, Cheng AG. Design multi-stopband laminate acoustic metamaterials for structural-acoustic coupled system. Mechanical Systems and Signal Processing 2019,115:418-433.

8. Figotin A, Vitebskiy I. Slow light in photonic crystals. Waves in Random \& Complex Media 2006,36:282-284.

9. Notomi M. Theory of light propagation in strongly modulated photonic crystals: Refractionlike behavior in the vicinity of the photonic band gap. Physical Review B 2000,62:10696.

10. Baena JD, Marqués R, Medina F, Martel J. Artificial magnetic metamaterial design by using spiral resonators. Physical Review B 2004,69: 014402.

11. Klein MW, Wegener M, Feth $\mathrm{N}$, Linden S. Experiments on second- and third-harmonic generation from magnetic metamaterials. Optics Express 2007,15:5238-5247.

12. Padilla WJ, Taylor AJ, Highstrete C, Lee M, Averitt RD. Dynamical Electric and Magnetic Metamaterial Response at Terahertz Frequencies. Physical Review Letters 2006,96:107401.

13. He ZC, Li E, Wang G, Li GY, Xia Z. Development of an efficient algorithm to analyze the elastic wave in acoustic metamaterials. Acta Mechanica 2016,227:1-16.

14. He ZC, Xiao X, Li E. Design for structural vibration suppression in laminate acoustic metamaterials. Composites Part B Engineering 2017,131:237-252.

15. Li E, He ZC, Hu JY, Long XY. Volumetric locking issue with uncertainty in the design of locally resonant acoustic metamaterials. Computer Methods in Applied Mechanics \& Engineering 2017,324:128-148.

16. Li E, He ZC, Wang G. An exact solution to compute the band gap in phononic crystals. Computational Materials Science 2016,122:72-85.

17. Li E, He ZC, Wang G, Liu GR. An ultra-accurate numerical method in the design of liquid phononic crystals with hard inclusion. Computational Mechanics 2017,60:1-14.

18. Xiaoming, Zhou, Xiaoning, Gengkai. Elastic metamaterials with local resonances: an overview. Theoretical and Applied Mechanics Letters 2012,2:1-12.

19. Zhu R, Huang GL, Huang HH, Sun CT. Experimental and numerical study of guided wave propagation in a thin metamaterial plate. Physics Letters A 2011,375(30):2863-2867.

20. Zhu R, Liu XN, Hu GK, Sun CT, Huang GL. A chiral elastic metamaterial beam for broadband vibration suppression. Journal of Sound \& Vibration 2014,333:2759-2773.

21. Zhu R, Liu XN, Hu GK, Yuan FG, Huang GL. Microstructural designs of plate-type elastic metamaterial and their potential applications: a review. International Journal of Smart \& Nano Materials 2015,6:14-40. 
22. Islam MT, Newaz G. Metamaterial with mass-stem array in acoustic cavity. Applied Physics Letters 2012,100:509.

23. Yang Z, Dai HM, Chan NH, Ma GC, Sheng P. Acoustic metamaterial panels for sound attenuation in the 50-1000 Hz regime. Applied Physics Letters 2010,96:1833-1821.

24. Ang LYL, Yong KK, Lee HP. Acoustic Metamaterials: A Potential for Cabin Noise Control in Automobiles and Armored Vehicles. International Journal of Applied Mechanics 2016,08:1650072.

25. Billon K, Zampetakis I, Scarpa F, Ouisse M, Sadoulet-Reboul E, Collet M, et al. Mechanics and band gaps in hierarchical auxetic rectangular perforated composite metamaterials. Composite Structures 2016,160: 1042-1050.

26. Liu Z, Rumpler R, Feng L. Broadband locally resonant metamaterial sandwich plate for improved noise insulation in the coincidence region. Composite Structures 2018, 200:165-172.

27. Shi HYY, Tay TE, Lee HP. Numerical studies on composite meta-material structure for mid to low frequency elastic wave mitigation. Composite Structures 2018, 195:136-146.

28. Song GY, Cheng Q, Huang B, Dong HY, Cui TJ. Broadband fractal acoustic metamaterials for low-frequency sound attenuation. Applied Physics Letters 2016, 109(13): 131901.

29. Wang X, Zhao H, Luo X, Huang Z. Membrane-constrained acoustic metamaterials for low frequency sound insulation. Applied Physics Letters 2016,108(4):1734.

30. Zhang S, Yin L, Fang N. Focusing ultrasound with an acoustic metamaterial network. Physical Review Letters 2009,102(19):194301.

31. Gonella S, To AC, Liu WK. Interplay between phononic bandgaps and piezoelectric microstructures for energy harvesting. Journal of the Mechanics \& Physics of Solids 2016,57:621-633.

32. Mikoshiba K, Manimala JM, Sun CT. Energy harvesting using an array of multifunctional resonators. Journal of Intelligent Material Systems \& Structures 2013,24:168-179.

33. Cselyuszka N, Sečujski M, Crnojević-Bengin V. Novel negative mass density resonant metamaterial unit cell. Physics Letters A 2015,379:33-36.

34. Assouar B, Oudich M, Zhou X. Acoustic metamaterials for sound mitigation. Comptes rendus Physique 2016,17:524-532.

35. Wang X. Dynamic behaviour of a metamaterial system with negative mass and modulus. International Journal of Solids \& Structures 2014,51:1534-1541.

36. Alamri S, Li B, Tan KT. Dynamic load mitigation using dissipative elastic metamaterials with multiple Maxwell-type oscillators. Journal of Applied Physics 2018,123:095111.

37. Khan MH, Li B, Tan KT. Impact Load Wave Transmission in Elastic Metamaterials. International Journal of Impact Engineering 2018,118:50-59.

38. Li B, Liu Y, Tan KT, Li B, Liu Y, Tan KT. A novel meta-lattice sandwich structure for dynamic load mitigation. Journal of Sandwich Structures \& Materials 2017:1-26.

39. Naify CJ, Chang CM, Mcknight G, Nutt S. Transmission loss of membrane-type acoustic metamaterials with coaxial ring masses. Journal of Applied Physics 2011,110:751.

40. Pai PF, Peng H, Jiang S. Acoustic metamaterial beams based on multi-frequency vibration absorbers. International Journal of Mechanical Sciences 2014,79:195-205.

41. Pope SA, Daley S. Viscoelastic locally resonant double negative metamaterials with controllable effective density and elasticity. Physics Letters A 2010,374:4250-4255. 
42. Huang GL, Sun CT. Band Gaps in a Multiresonator Acoustic Metamaterial. Journal of Vibration \& Acoustics 2010,132:1015-1016.

43. Lakes RS, Lee T, Bersie A, Wang YC. Extreme damping in composite materials with negative-stiffness inclusions. Nature 2001,410:565-567.

44. Tan KT, Huang $\mathrm{HH}$, Sun CT. Optimizing the band gap of effective mass negativity in acoustic metamaterials. Applied Physics Letters 2012,101 (24):3966.

45. Tan KT, Huang $\mathrm{HH}$, Sun CT. Blast-wave impact mitigation using negative effective mass density concept of elastic metamaterials. International Journal of Impact Engineering 2014,64:20-29.

46. Chen H, Li XP, Chen YY, Huang GL. Wave propagation and absorption of sandwich beams containing interior dissipative multi-resonators. Ultrasonics 2016,76:99-108.

47. Nouh M, Aldraihem O, Baz A. Wave propagation in metamaterial plates with periodic local resonances. Journal of Sound \& Vibration 2015,341:53-73.

48. Chen YY, Barnhart MV, Chen JK, Hu GK, Sun CT, Huang GL. Dissipative elastic metamaterials for broadband wave mitigation at subwavelength scale. Composite Structures 2016,136:358-371.

49. Barnhart MV, Xianchen X, Yangyang C, Shun Z, Jizhou S, Guoliang H. Experimental demonstration of a dissipative multi-resonator metmaterial for broadband elastic wave attenuation. Journal of Sound and Vibration 2019, 438:1-12.

50. Merheb B, Deymier PA, Muralidharan K, Bucay J, Jain M, Aloshynalesuffleur M, et al. Viscoelastic effect on acoustic band gaps in polymer-fluid composites. Modelling \& Simulation in Materials Science \& Engineering 2015,17(7):75013-75013.

51. Hussein MI, Frazier MJ. Metadamping: An emergent phenomenon in dissipative metamaterials. Journal of Sound \& Vibration 2013,332:4767-4774.

52. Zapfe J, Lesieutre G. Broadband vibration damping in beams using distributed viscoelastic tuned mass absorbers. In: Structure, Structural Dynamics and Materials Conference; 2013.

53. Thompson DJ. A continuous damped vibration absorber to reduce broad-band wave propagation in beams. Journal of Sound \& Vibration 2008,311:824-842.

54. Carrara M, Ruzzene M. High stiffness, high damping chiral metamaterial assemblies for low-frequency applications. In: Health Monitoring of Structural and Biological Systems; 2013.

55. Li QQ, Song K, He ZC, Li E, Cheng AG, Chen T. The artificial tree (AT) algorithm. Engineering Applications of Artificial Intelligence 2017,65:99-110. 